TRANSACTIONS OF THE

AMERICAN MATHEMATICAL SOCIETY

Volume 364, Number 6, June 2012, Pages 2847-2882

S 0002-9947(2012)05376-1

Article electronically published on January 31,2012

\title{
GROUPS WITH FREE REGULAR LENGTH FUNCTIONS IN $\mathbb{Z}^{n}$
}

\author{
OLGA KHARLAMPOVICH, ALEXEI MYASNIKOV, VLADIMIR REMESLENNIKOV, \\ AND DENIS SERBIN
}

\begin{abstract}
This is the first paper in a series of three where we take on the unified theory of non-Archimedean group actions, length functions and infinite words. Our main goal is to show that group actions on $\mathbb{Z}^{n}$-trees give one a powerful tool to study groups. All finitely generated groups acting freely on $\mathbb{R}$-trees also act freely on some $\mathbb{Z}^{n}$-trees, but the latter ones form a much larger class. The natural effectiveness of all constructions for $\mathbb{Z}^{n}$-actions (which is not the case for $\mathbb{R}$-trees) comes along with a robust algorithmic theory. In this paper we describe the algebraic structure of finitely generated groups acting freely and regularly on $\mathbb{Z}^{n}$-trees and give necessary and sufficient conditions for such actions.
\end{abstract}

\section{Contents}

1. Introduction

2. Length functions, actions, infinite words

2.1. Lyndon length functions and free actions 2850

2.2. Regular length functions 2851

2.3. Regular actions 2853

2.4. Infinite words and length functions 2855

3. Commutation in infinite words 2857

4. Complete $\mathbb{Z}^{n}$-free groups 2860

4.1. Elementary transformations of infinite words 2861

4.2. Minimal sets of generators and pregroups 2864

4.3. Algebraic structure of complete $\mathbb{Z}^{n}$-free groups $\quad 2866$

5. Regular free actions of HNN extensions 2869

5.1. Cyclically reduced centralizers and attached elements 2869

5.2. Connecting elements 2871

5.3. Main construction 2872

References $\quad 2880$

\section{INTRODUCTION}

This is the first paper in a series of three where we take on the unified theory of non-Archimedean group actions, length functions and infinite words. Our main goal is to show that group actions on $\mathbb{Z}^{n}$-trees give one a powerful tool to study groups. All finitely generated groups acting freely on $\mathbb{R}$-trees also act freely on some $\mathbb{Z}^{n}$-trees, but the latter ones form a much larger class. The natural effectiveness of all constructions for $\mathbb{Z}^{n}$-actions (which is not the case for $\mathbb{R}$-trees) comes along with

Received by the editors August 9, 2009 and, in revised form, March 22, 2010 and May 3, 2010. 2010 Mathematics Subject Classification. Primary 20E08, 20F65. 
a robust algorithmic theory. In this paper we describe the algebraic structure of finitely generated groups acting freely and regularly on $\mathbb{Z}^{n}$-trees and give necessary and sufficient conditions for such actions.

Group actions and length functions. There are two classical approaches to the fundamental groups of graphs of groups: one uses group actions on trees, and another one - length functions.

In his seminal book [39] Serre laid down fundamentals of the theory of groups acting freely on simplicial trees. In the following decade Serre's approach unified several geometric and combinatorial methods of group theory into a unique tool, known today as Bass-Serre theory. This was one of the major achievements of combinatorial group theory in the 1970s.

In the 1980s Morgan and Shalen introduced group actions on $\Lambda$-trees for an arbitrary ordered abelian group $\Lambda$ 30. In particular, they studied actions on $\mathbb{R}$ trees in relation with Thurston's Geometrization Theorem.

Alperin and Bass [1] developed the initial framework of the theory of group actions on $\Lambda$-trees and stated the fundamental research goals: find the group theoretic information carried by an action (by isometries) on a $\Lambda$-tree; generalize Bass-Serre theory to actions on arbitrary $\Lambda$-trees.

A joint effort of several researchers culminated in a description of finitely generated groups acting freely on $\mathbb{R}$-trees [4, 10, which is now known as Rips' theorem: a finitely generated group acts freely on an $\mathbb{R}$-tree if and only if it is a free product of free abelian groups and surface groups (with the exception of non-orientable groups of genus 1,2 , and 3). The key ingredient of this theory is the so-called "Rips machine", the idea of which comes from Makanin's algorithm for solving equations in free groups [28. The Rips machines appear in applications as a general tool that takes a sequence of isometric actions of a group $G$ on some "negatively curved spaces" and produces an isometric action of $G$ on an $\mathbb{R}$-tree in the GromovHausdorff limit. Free actions on $\mathbb{R}$-trees cover all Archimedean actions, since every group acting freely on a $\Lambda$-tree for an Archimedean ordered abelian group $\Lambda$ also acts freely on an $\mathbb{R}$-tree.

The case of non-Archimedean free actions is wide open. In 3] Bass studied finitely generated groups acting freely on $(\Lambda \oplus \mathbb{Z})$-trees with respect to the lexicographic order on $\Lambda \oplus \mathbb{Z}$. Recently, Guirardel (see [13) studied finitely generated groups acting freely on an $\mathbb{R}^{n}$-tree (with the lexicographic order). However, the following main problem of the Alperin-Bass program remains largely intact.

Problem. Describe finitely presented (finitely generated) groups acting freely on an arbitrary $\Lambda$-tree.

Much earlier Lyndon introduced groups equipped with length functions with values in $\mathbb{Z}$ as a tool to carry over Nielsen cancellation theory from free groups to a much more general setting [26] (see also [27]). Some partial results (for $\Lambda=\mathbb{Z}$ or $\Lambda=\mathbb{R}$ ) were obtained in [15, 16, 14, 35, 2, . In [5] Chiswell described a crucial construction that shows that a group with a length function with values in $\mathbb{R}$ comes naturally from some action of the group on an $\mathbb{R}$-tree, and vice versa. It was realized later that a similar construction holds for an arbitrary group with a free Lyndon length function with values in $\Lambda$ [30]. Thus free group actions and free Lyndon length functions are just two equivalent languages describing the same objects. We refer to the book [6] for a detailed discussion on the subject. 
Nowadays, the geometric method of group actions seems much more in use than the length functions, even though the Nielsen theory still gives very powerful results [17, 18, 41, 42, 8, 11]. There are several reasons for it. First, the Lyndon's abstract axiomatic approach to length functions is less intuitive than group actions. Second, the current development of the abstract Nielsen theory is incomplete and insufficient - one of the principal notions of complete actions and regular length functions was not in use until recently (see the discussion below). Third, and this is crucial, an abstract analog of the Rips machine for length functions with values in $\mathbb{R}$ (or an arbitrary $\Lambda$ ) was not introduced or developed enough to be robust in applications. Notice that in the case of $\Lambda=\mathbb{Z}$ the completeness and regularity come almost for free, so there was no need for axiomatic formalization - it went mostly unnoticed. The regularity axiom appeared first in [31, 32, as a tool to deal with length functions in $\mathbb{Z}^{n}$ (with respect to the lexicographic order). On the other hand, an analog of the Rips machine for $\Lambda=\mathbb{Z}$ did exist for a long time - the original Makanin-Razborov process [36] for solving equations in free groups. But it was not recognized as such until [20, 21, where it was used systematically to get splittings of groups.

Motivation and recent progress. Introduction of infinite $\Lambda$-words was one of the major recent developments in the theory of group actions (see Section 2.4 for definitions). In 32 Myasnikov, Remeslennikov and Serbin showed that groups admitting faithful representations by $\Lambda$-words act freely on some $\Lambda$-trees, while Chiswell proved the converse [7]. This gives another equivalent approach to group actions. Now one can bypass the axiomatic viewpoint on length functions and work instead with $\Lambda$-words in the same manner as with the ordinary words in the standard free groups, which is much more intuitive and practical. In particular, one can deal with subgroups of groups acting freely on $\mathbb{Z}^{n}$-trees (for example, fully residually free groups) using the standard Stallings' folding argument [32, 33, 23, 34.

Formulation of the regularity axiom, discussed above, is another important ingredient of the theory of group actions. This axiom ensures that the group is complete with respect to Gromov's inner product, i.e., Gromov's product of two elements of the group can be realized as the length of a particular element of the group (not only as an abstract length). In the language of actions this means that all branch points of the group action are in one same orbit, as well as the base point. This allows one to use the Nielsen cancellation argument at full strength, very similar to the case of a free group. The regularity (completeness) condition is crucial for the existence of Makanin-Razborov type processes over groups with $\Lambda$-length functions. We discuss this in the third paper of the series for $\Lambda=\mathbb{Z}^{n}$, while in the second one we show that every group acting freely on a $\mathbb{Z}^{n}$-tree isometrically embeds into a group with a regular action on some $\mathbb{Z}^{m}$-tree.

Results. In Section 2 we recall the notions of length functions, infinite words, and discuss the regularity axiom. Here we provide some crucial examples of groups with regular length functions. In particular, we show that every finitely generated group acting freely on an $\mathbb{R}$-tree has a free regular length function in $\mathbb{Z}^{n}$. More generally, we show that certain types of HNN extensions have free regular length functions that extend a given regular free length function on the base groups. Notice that these are precisely the HNN extensions that play the central role in the elimination process from 20]. In Section 2.3 we give an equivalent geometric characterization 
of group actions that come from the regular length functions. This geometric characterization plays an important part in the whole theory of group actions on trees.

Commutation of $\mathbb{Z}^{n}$-words is an important technical instrument, which can be viewed as a "non-standard" version of the commutation in free groups; we discuss it in Section 3 ,

In Section 4 we describe the algebraic structure of a finitely generated group $G$ with a regular length function in $\mathbb{Z}^{n}$ in terms of HNN extensions of a very particular type. To this end, in Section 4.1 we introduce Nielsen-like moves and show that every finite set of generators of $G$ can be transformed by a sequence of elementary moves into a minimal one (relative to the $\Lambda$-length). Then, in Section 4.2 we show that any minimal finite set of generators of $G$ gives an HNN-splitting of $G$. Here we use the technique of Stallings' pregroups and their universal groups [40]. This is an analog of the celebrated Nielsen theorem on subgroups of free groups. Interestingly, the whole approach closely resembles the Gröbner basis method and Buchberger's algorithm. Theorem 7 in Section 4.3 gives the description of the algebraic structure of $G$ in terms of a finite sequence of HNN extensions of a certain type. In Section 5 we prove that the converse of Theorem 7 also holds, thus finishing the complete description of groups acting freely and regularly on $\mathbb{Z}^{n}$-trees.

\section{Length FunCtions, ACTIONS, INFInite WORDS}

Here we introduce basic definitions and notation which are to be used throughout the whole paper.

2.1. Lyndon length functions and free actions. Let $G$ be a group and $A$ an ordered abelian group. Then a function $l: G \rightarrow A$ is called a (Lyndon) length function on $G$ if the following conditions hold:

(L1) $\forall g \in G: l(g) \geqslant 0$ and $l(1)=0$;

(L2) $\forall g \in G: l(g)=l\left(g^{-1}\right)$;

(L3) $\forall g, f, h \in G: c(g, f)>c(g, h) \rightarrow c(g, h)=c(f, h)$, where $c(g, f)=$ $\frac{1}{2}\left(l(g)+l(f)-l\left(g^{-1} f\right)\right)$.

Sometimes we refer to length functions with values in $A$ as $A$-length functions. Observe that the standard length function on a finitely generated group (with respect to a fixed finite generating set) is not necessarily a Lyndon length function. In this paper all length functions are Lyndon ones.

Notice that $c(g, f)$ may not be defined in $A$ (if $l(g)+l(f)-l\left(g^{-1} f\right)$ is not divisible by 2 ), so in the axiom (L3) we assume that $A$ is canonically embedded into a divisible ordered abelian group $A_{\mathbb{Q}}=A \otimes_{\mathbb{Z}} \mathbb{Q}$ (see [32] for details).

It is not difficult to derive the following two properties of length functions from the axioms (L1)-(L3):

- $\forall g, f \in G: l(g f) \leqslant l(g)+l(f)$;

- $\forall g, f \in G: 0 \leqslant c(g, f) \leqslant \min \{l(g), l(f)\}$.

A length function $l: G \rightarrow A$ is called free if it satisfies the following two axioms:

(L4) $\forall g, f \in G: c(g, f) \in A$.

(L5) $\forall g \in G: g \neq 1 \rightarrow l\left(g^{2}\right)>l(g)$.

As we have mentioned in the introduction group actions on $A$-trees provide a geometric counterpart to $A$-length functions. To explain we need the following definitions, which we also use later in the text. 
Let $X$ be a non-empty set, and let $A$ be an ordered abelian group. An $A$-metric on $X$ is a mapping $p: X \times X \longrightarrow A$ such that for all $x, y, z \in X$ :

(M1) $p(x, y) \geq 0$ and $p(x, y)=0$ if and only if $x=y$,

(M2) $p(x, y)=p(y, x)$,

(M3) $p(x, y) \leq p(x, z)+p(y, z)$.

In this case the pair $(X, p)$ is an $A$-metric space. If $(X, p)$ and $\left(X^{\prime}, p^{\prime}\right)$ are $A$ metric spaces, an isometry from $(X, p)$ to $\left(X^{\prime}, p^{\prime}\right)$ is a mapping $f: X \rightarrow X^{\prime}$ such that $p(x, y)=p^{\prime}(f(x), f(y))$ for all $x, y \in X$.

For elements $a, b \in A$ the closed segment $[a, b]$ is defined by

$$
[a, b]=\{c \in A \mid a \leqslant c \leqslant b\} .
$$

More generally, a segment in an $A$-metric space is the image of an isometry $\alpha$ : $[a, b] \rightarrow X$ for some $a, b \in A$. The endpoints of the segment are $\alpha(a), \alpha(b)$. A segment with endpoints $x, y \in X$ is denoted by $[x, y]$. An $A$-metric space $(X, p)$ is geodesic if for all $x, y \in X$ there is a segment in $X$ with endpoints $x, y$.

An $A$-tree is an $A$-metric space $(X, p)$ such that:

(T1) $(X, p)$ is geodesic,

(T2) if two segments of $(X, p)$ intersect in a single point, which is an endpoint of both, then their union is a segment,

(T3) the intersection of two segments with a common endpoint is also a segment.

We say that a group $G$ acts on an $A$-tree $X$ if it acts on $X$ by isometries, i.e., an embedding of $G$ into the group of isometries of $X$ is fixed. An action of $G$ on $X$ is termed free if for every $1 \neq g \in G$ neither $g$ nor $g^{2}$ has a fixed point in $X$.

The following fundamental result relates free actions with free Lyndon length functions.

Theorem 1 ([6], 30]). A group $G$ has a free Lyndon length function with values in $A$ if and only if $G$ acts freely on an A-tree.

If a group $G$ acts freely on an $A$-tree $X$, then the length function on $G$ based at $x$, defined as $L_{x}(g)=d(x, g x), g \in G$, is a free Lyndon length function on $G$.

For elements $g_{1}, \ldots, g_{n} \in G$ we write

$$
g=g_{1} \circ \cdots \circ g_{n}
$$

if $g=g_{1} \cdots g_{n}$ and $l(g)=l\left(g_{1}\right)+\cdots+l\left(g_{n}\right)$. Also, for $\alpha \in A$ we write $g=g_{1} \circ_{\alpha} g_{2}$ if $g=g_{1} g_{2}$ and $c\left(g_{1}^{-1}, g_{2}\right)<\alpha$.

2.2. Regular length functions. In this section we define regular length functions and show some examples of groups with regular length functions. Theorem 8 gives a host of new examples of such groups. In the subsequent paper [24] we discuss a geometric characterization of groups with regular length functions.

A length function $l: G \rightarrow A$ is called regular if it satisfies the regularity axiom:

(L6) $\forall g, f \in G, \exists u, g_{1}, f_{1} \in G$ :

$$
g=u \circ g_{1} \& f=u \circ f_{1} \& l(u)=c(g, f) .
$$

Here are several examples of groups with regular free length functions.

Example 1. Let $F=F(X)$ be a free group on $X$. The length function

$$
\text { | } \mid: F \rightarrow \mathbb{Z}
$$

where $|f|$ is the length of $f \in F$ as a word in $X^{ \pm 1}$, is regular. 
The following is a more general example.

Example 2. Let $F=F(X)$ be a free group on $X, H$ a finitely generated subgroup of $H$, and $l_{H}$ the restriction to $H$ of the length function in $F$ relative to $X$. Then $l_{H}$ is a regular length function on $H$ if and only if there exists a basis $U$ of $H$ such that every two non-equal elements from $U^{ \pm 1}$ have different initial letters.

Proof. The straightforward proof is not very hard. On the other hand, one can argue using the transformations $\mu$ and $\nu$ from Section 4.1. Indeed, any reduced generating set $U$ of $H$ satisfies the conditions above, since the transformations $\mu$ and $\nu$ do not reduce the total length. Conversely, if a generating set $U$ satisfies the conditions, then there is no cancellation in the products $u v$ for $u, v \in U^{ \pm 1}, u \neq v$, and the result follows as in Example 1

Example 3 ([32]). Lyndon's free $\mathbb{Z}[t]$-group $F^{\mathbb{Z}[t]}$ has a regular free length function with values in $\mathbb{Z}[t]$.

The next example is just a particular case of Theorem 8 below.

Example 4. Let $F=F(X)$ be a free group with basis $X,||$ the standard length function on $F$ relative to $X$, and $u, v \in F$ such that $|u|=|v|$ and $u$ is not conjugate to $v^{-1}$. Then the HNN extension,

$$
G=\left\langle F, s \mid u^{s}=v\right\rangle
$$

has a regular free length function $l: G \rightarrow \mathbb{Z}^{2}$ which extends $|\cdot|$.

Example 5. For any $n \geq 1$ the orientable surface group

$$
G=\left\langle x_{1}, x_{2}, \ldots, x_{2 n-1}, x_{2 n} \mid\left[x_{1}, x_{2}\right] \cdots\left[x_{2 n-1}, x_{2 n}\right]=1\right\rangle
$$

has a regular free length function $l: G \rightarrow \mathbb{Z}^{2}$.

Proof. It suffices to represent $G$ as an HNN extension from Example 4 . The word

$$
R(X)=x_{1} \cdots x_{2 n} x_{1}^{-1} \cdots x_{2 n}^{-1}
$$

is quadratic, so there exists an automorphism $\phi$ of $F=F\left(x_{1}, \ldots, x_{2 n}\right)$ such that

$$
R(X)^{\phi}=\left[x_{1}, x_{2}\right] \cdots\left[x_{2 n-1}, x_{2 n}\right]
$$

(see, for example, Proposition 7.6 of [27]). It follows that $G$ is isomorphic to

$$
G^{\prime}=\left\langle x_{1}, \ldots, x_{2 n} \mid x_{1} \cdots x_{2 n} x_{1}^{-1} \cdots x_{2 n}^{-1}=1\right\rangle,
$$

which can be represented as an HNN extension of the required form

$$
G^{\prime}=\left\langle F\left(x_{2}, \ldots, x_{2 n}\right), x_{1} \mid x_{1}\left(x_{2} \cdots x_{2 n}\right) x_{1}^{-1}=x_{2 n} x_{2 n-1} \cdots x_{2}\right\rangle,
$$

since $\left|x_{2} \cdots x_{2 n}\right|=\left|x_{2 n} x_{2 n-1} \cdots x_{2}\right|$.

Example 6. For any $n, n \geq 3$, the non-orientable surface group

$$
G=\left\langle x_{1}, x_{2}, \ldots, x_{n} \mid x_{1}^{2} x_{2}^{2} \cdots x_{n}^{2}=1\right\rangle
$$

has a regular free length function $l: G \rightarrow \mathbb{Z}^{2}$.

Proof. Again, it suffices to represent $G$ as an HNN extension from Example 4

An argument similar to the one in the proof of Example 5] shows that the group $G$ is isomorphic to

$$
G^{\prime}=\left\langle x_{1}, x_{2}, \ldots, x_{n} \mid x_{1} \cdots x_{n-1} x_{n} x_{1}^{-1} \cdots x_{n-1}^{-1} x_{n}\right\rangle,
$$


and the result follows, since the presentation above can be written as

$$
G^{\prime}=\left\langle x_{1}, x_{2}, \ldots, x_{n} \mid x_{1}\left(x_{2} \cdots x_{n-1} x_{n}\right) x_{1}^{-1}=x_{n}^{-1} x_{n-1} \cdots x_{2}\right\rangle .
$$

Example 7. A free abelian group of rank $n$ has a free regular length function in $\mathbb{Z}^{n}$.

Proof. Let $G=\mathbb{Z}^{n}$ be a free abelian group of rank $n$. Then $G$ is an ordered abelian group relative to the right lexicographic order $\leq$. The absolute value $|u|$ of an element $u \in G$, defined as $|u|=\max \{u,-u\}$, gives a free length function $l: G \rightarrow \mathbb{Z}^{n}$. It is easy to see that $l$ is regular.

Example 8. Let $G_{i}, i=1,2$, be a group with a free regular length function $l_{i}: G_{i} \rightarrow \mathbb{Z}^{n}$. Then the free product $G=G_{1} * G_{2}$ has a free regular length function in $l: G \rightarrow \mathbb{Z}^{n}$ that extends the functions $l_{1}$ and $l_{2}$.

Proof. Let $g \in G$ be given in the reduced form $g=u_{1} v_{1} \cdots u_{k} v_{k}$, where $u_{1}, \ldots, u_{k} \in$ $G_{1}$ and $v_{1}, \ldots, v_{k} \in G_{2}$. Define $l: G \rightarrow \mathbb{Z}^{n}$ by

$$
l(g)=\sum_{i=1}^{k}\left(l_{1}\left(u_{i}\right)+l_{2}\left(v_{i}\right)\right) .
$$

$l$ is a free length function (see, for example, 6]). Also, note that $c(g, h)=0$ if $g \in G_{1}$ and $h \in G_{2}$. Next, $l$ is regular. Indeed, let $g=g_{1} \cdots g_{k}, h=h_{1} \cdots h_{n} \in G$, where the products $g_{1} \cdots g_{k}, h_{1} \cdots h_{n}$ are reduced and $k \leq n$. Let $m=\max \left\{i \mid g_{i}=h_{i}\right\}$. If $m=k$, then $h=g \circ\left(h_{m+1} \cdots h_{n}\right)$ and $c(g, h)=l(g)$, so the regularity axiom holds for the pair $g, h$. If $m=n$, then necessarily $m=k$ and $h=g$, so the axiom holds again. Now assume that $m<k$. If we denote $c=g_{1} \cdots g_{m}$, then $g=$ $c \circ g_{m+1} \circ g^{\prime}, h=c \circ h_{m+1} \circ h^{\prime}$, where $g^{\prime}=g_{m+2} \cdots g_{k}, h^{\prime}=h_{m+2} \cdots h_{n}\left(g^{\prime}\right.$ is trivial if $k=m+1$, and $h^{\prime}$ is trivial if $\left.n=m+1\right)$. Hence, $g^{-1} h=\left(g^{\prime}\right)^{-1} \circ\left(g_{m+1}^{-1} h_{m+1}\right) \circ h^{\prime}$ and

$$
\begin{gathered}
c(g, h)=\frac{1}{2}\left(l(g)+l(h)-l\left(g^{-1} h\right)\right)=\frac{1}{2}\left(l(c)+l\left(g_{m+1}\right)+l\left(g^{\prime}\right)+l(c)+l\left(h_{m+1}\right)+l\left(h^{\prime}\right)\right. \\
\left.-\left(l\left(g^{\prime}\right)+l\left(g_{m+1}^{-1} h_{m+1}\right)+l\left(h^{\prime}\right)\right)\right)=l(c)+c\left(g_{m+1}, h_{m+1}\right) .
\end{gathered}
$$

Since both $g_{m+1}$ and $h_{m+1}$ belong to the same factor $G_{i}$ and the length function on $G_{i}$ is regular, it follows that there exists $u \in G_{i}$ such that $l(u)=c\left(g_{m+1}, h_{m+1}\right)$, $g_{m+1}=u \circ v, h_{m+1}=u \circ w$ for some $v, w \in G_{i}$. Hence, $g=(c u) \circ v \circ g^{\prime}, h=$ $(c u) \circ w \circ h^{\prime}$, where $c(g, h)=l(c u)$ and $c u \in G$.

Example 9. Let $G$ be a finitely generated group acting freely on an $\mathbb{R}$-tree. Then $G$ has a free regular length function in $\mathbb{Z}^{n}$, where $n$ is the maximal rank of free abelian subgroups (centralizers) of $G$.

Proof. By Rips' theorem every finitely generated group acting freely on an $\mathbb{R}$-tree is a free product of groups described in Examples [5, 6, and 7] hence the result.

2.3. Regular actions. In this section we give a geometric characterization of group actions that come from the regular length functions.

Definition 1. Let $G$ act on a $\Lambda$-tree $\Gamma$. The action is regular with respect to $x \in \Gamma$ if for any $g, h \in G$ there exists $f \in G$ such that $[x, f x]=[x, g x] \cap[x, h x]$.

The next lemma shows that regular actions exactly correspond to regular length functions (hence the term). 
Lemma 1. Let $G$ act on a $\Lambda$-tree $\Gamma$. Then the action of $G$ is regular with respect to $x \in \Gamma$ if and only if the length function $L_{x}: G \rightarrow \Lambda$ based at $x$ is regular.

Proof. Let $d$ be the $\Lambda$-metric on $\Gamma$. By definition, the length function $L_{x}$ is regular if for every $g, h \in G$ there exists $f \in G$ such that $g=f g_{1}, h=f h_{1}$, where $L_{x}(f)=c(g, h)$ and $L_{x}(g)=L_{x}(f)+L_{x}\left(g_{1}\right), L_{x}(h)=L_{x}(f)+L_{x}\left(h_{1}\right)$.

Suppose the action of $G$ is regular with respect to $x$. Then for $g, h \in G$ there exists $f \in G$ such that $[x, f x]=[x, g x] \cap[x, h x]$. We have $[x, g x]=[x, f x] \cup$ $[f x, g x],[x, h x]=[x, f x] \cup[f x, h x]$ and $d(f x, g x)=d\left(x,\left(f^{-1} g\right) x\right)=L_{x}\left(f^{-1} g\right)$, $d(f x, h x)=d\left(x,\left(f^{-1} h\right) x\right)=L_{x}\left(f^{-1} h\right)$. Taking $g_{1}=f^{-1} g, h_{1}=f^{-1} h$ we have $L_{x}(g)=L_{x}(f)+L_{x}\left(g_{1}\right), L_{x}(h)=L_{x}(f)+L_{x}\left(h_{1}\right)$. Finally, since $c(g, h)=\frac{1}{2}\left(L_{x}(g)+\right.$ $\left.L_{x}(h)-L_{x}\left(g^{-1} h\right)\right)$ and $L_{x}\left(g^{-1} h\right)=d\left(x,\left(g^{-1} h\right) x\right)=d(g x, h x)=d(f x, g x)+$ $d(f x, h x)$ we get $L_{x}(f)=c(g, h)$.

Suppose that $L_{x}$ is regular. Then from $g=f g_{1}, h=f h_{1}$, where $L_{x}(g)=L_{x}(f)+$ $L_{x}\left(g_{1}\right), L_{x}(h)=L_{x}(f)+L_{x}\left(h_{1}\right)$, it follows that $[x, g x]=[x, f x] \cup[f x, g x],[x, h x]=$ $[x, f x] \cup[f x, h x]$. Now, $L_{x}(f)=c(g, h)=\frac{1}{2}\left(L_{x}(g)+L_{x}(h)-L_{x}\left(g^{-1} h\right)\right)$, so $2 d(x, f x)=d(x, g x)+d(x, h x)-d\left(x,\left(g^{-1} h\right) x\right)=d(x, g x)+d(x, h x)-d(g x, h x)$. In other words,

$$
\begin{gathered}
d(g x, h x)=d(x, g x)+d(x, h x)-2 d(x, f x)=(d(x, g x)-d(x, f x)) \\
+(d(x, h x)-d(x, f x))=d(f x, g x)+d(f x, h x),
\end{gathered}
$$

which is equivalent to $[x, f x]=[x, g x] \cap[x, h x]$.

Lemma 2. Let $G$ act minimally on a $\Lambda$-tree $\Gamma$. If the action of $G$ is regular with respect to $x \in \Gamma$, then all branch points of $\Gamma$ are $G$-equivalent.

Proof. From minimality of the action it follows that $\Gamma$ is spanned by the set of points $G x=\{g x \mid g \in G\}$.

Now let $y$ be a branch point in $\Gamma$. It follows that there exist (not unique in general) $g, h \in G$ such that $[x, y]=[x, g x] \cap[x, h x]$. From regularity of the action it follows that there exists $f \in G$ such that $y=f x$. Hence, every branch point is $G$-equivalent to $x$ and the statement of the lemma follows.

Lemma 3. Let $G$ act on a $\Lambda$-tree $\Gamma$. If the action of $G$ is regular with respect to $x \in \Gamma$, then it is regular with respect to any $y \in G x$.

Proof. We have to show that for every $g, h \in G$ there exists $f \in G$ such that $[y, f y]=[y, g y] \cap[y, h y]$. Since $y=t x$ for some $t \in G$, then we have to prove that $[t x,(f t) x]=[t x,(g t) x] \cap[t x,(h t) x]$. The latter equality is equivalent to $\left[x,\left(t^{-1} f t\right) x\right]=\left[x,\left(t^{-1} g t\right) x\right] \cap\left[x,\left(t^{-1} h t\right) x\right]$, which follows from regularity of the action with respect to $x$.

Lemma 4. Let $G$ act freely on a $\Lambda$-tree $\Gamma$ so that all branch points of $\Gamma$ are $G$ equivalent. Then the action of $G$ is regular with respect to any branch point in $\Gamma$.

Proof. Let $x$ be a branch point in $\Gamma$ and $g, h \in G$. If $g=h$, then $[x, g x] \cap[x, h x]=$ $[x, g x]$ and $g$ is the required element. Suppose $g \neq h$. Since the action is free, then $g x \neq h x$, and we consider the tripod formed by $x, g x, h x$. Hence, $y=Y(x, g x, h x)$ is a branch point in $\Gamma$, and by the assumption there exists $f \in G$ such that $y=f x$. 


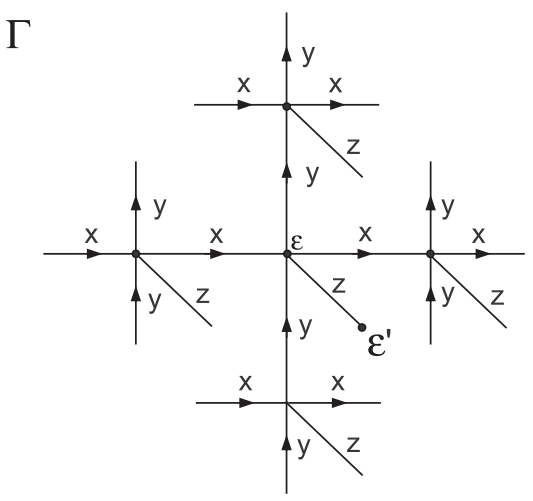

Figure 1. $\Gamma$ in Example 10.

Example 10. Let $\Gamma^{\prime}$ be the Cayley graph of a free group $F(x, y)$ with the basepoint $\epsilon$. Let $\Gamma$ be obtained from $\Gamma^{\prime}$ by adding an edge labeled by $z \neq x^{ \pm 1}, y^{ \pm 1}$ at every vertex of $\Gamma^{\prime} . F(x, y)$ has a natural action on $\Gamma^{\prime}$ which we can extend to the action on $\Gamma$. The edge at $\epsilon$ labeled by $z$ has an endpoint not equal to $\epsilon$, and we denote it by $\epsilon^{\prime}$. Observe that the action of $F(x, y)$ on $\Gamma$ is regular with respect to $\epsilon$ but is not regular with respect to $\epsilon^{\prime}$.

2.4. Infinite words and length functions. In this subsection at first we recall some notions from the theory of ordered abelian groups (for all the details refer to the books [12] and 25]) and then following [32] describe the construction of infinite words.

Let $A$ be an ordered abelian group. Recall that for elements $a, b \in A$ the closed segment $[a, b]$ is defined by

$$
[a, b]=\{c \in A \mid a \leqslant c \leqslant b\} .
$$

Now a subset $C \subset A$ is called convex if for every $a, b \in C$ the set $C$ contains $[a, b]$. In particular, a subgroup $B$ of $A$ is convex if $[0, b] \subset B$ for every positive $b \in B$.

An ordered abelian group $A$ is called discretely ordered if $A$ has a minimal positive element (we denote it by $1_{A}$ ). In this event, for any $a \in A$ the following hold:

1) $a+1_{A}=\min \{b \mid b>a\}$,

2) $a-1_{A}=\max \{b \mid b<a\}$.

Observe that if $A$ is any ordered abelian group, then $\mathbb{Z} \oplus A$ is discretely ordered with respect to the right lexicographic order.

Let $A$ be a discretely ordered abelian group and let $X=\left\{x_{i} \mid i \in I\right\}$ be a set. Put $X^{-1}=\left\{x_{i}^{-1} \mid i \in I\right\}$ and $X^{ \pm}=X \cup X^{-1}$. An $A$-word is a function of the type

$$
w:\left[1_{A}, \alpha_{w}\right] \rightarrow X^{ \pm}
$$

where $\alpha_{w} \in A, \alpha_{w} \geqslant 0$. The element $\alpha_{w}$ is called the length $|w|$ of $w$.

By $W(A, X)$ we denote the set of all $A$-words. Observe that $W(A, X)$ contains an empty $A$-word which we denote by $\varepsilon$.

Concatenation $u v$ of two $A$-words $u, v \in W(A, X)$ is an $A$-word of length $|u|+|v|$ and such that

$$
(u v)(a)= \begin{cases}u(a) & \text { if } 1_{A} \leqslant a \leqslant|u| \\ v(a-|u|) & \text { if }|u|<a \leqslant|u|+|v| .\end{cases}
$$


An $A$-word $w$ is reduced if $w\left(\beta+1_{A}\right) \neq w(\beta)^{-1}$ for each $1_{A} \leqslant \beta<|w|$. We denote by $R(A, X)$ the set of all reduced $A$-words. Clearly, $\varepsilon \in R(A, X)$.

Of course, concatenation $u v$ of two reduced words $u, v$ may not be reduced. We write $u \circ v$ instead of $u v$ in the case when $u v$ is reduced, i.e. $u(|u|) \neq v\left(1_{A}\right)^{-1}$. Obviously, o satisfies the following cancellation conditions:

$$
x \circ y=x \circ z \Longrightarrow y=z, \quad y \circ x=z \circ x \Longrightarrow y=z .
$$

If $w=w_{1} \circ u \circ w_{2}$, then $u$ is called a subword of $w$.

For $u \in W(A, X)$ and $\beta \in\left[1_{A}, \alpha_{u}\right]$ by $u_{\beta}$ we denote the restriction of $u$ on $\left[1_{A}, \beta\right]$. If $u \in R(A, X)$ and $\beta \in\left[1_{A}, \alpha_{u}\right]$, then

$$
u=u_{\beta} \circ \tilde{u}_{\beta}
$$

for some uniquely defined $\tilde{u}_{\beta}$.

An element $\operatorname{com}(u, v) \in R(A, X)$ is called the (longest) common initial segment of $A$-words $u$ and $v$ if

$$
u=\operatorname{com}(u, v) \circ \tilde{u}, \quad v=\operatorname{com}(u, v) \circ \tilde{v}
$$

for some (uniquely defined) $A$-words $\tilde{u}, \tilde{v}$ such that $\tilde{u}\left(1_{A}\right) \neq \tilde{v}\left(1_{A}\right)$.

Now, we can define the product of two $A$-words. Let $u, v \in R(A, X)$. If $\operatorname{com}\left(u^{-1}, v\right)$ is defined, then

$$
u^{-1}=\operatorname{com}\left(u^{-1}, v\right) \circ \tilde{u}, \quad v=\operatorname{com}\left(u^{-1}, v\right) \circ \tilde{v}
$$

for some uniquely defined $\tilde{u}$ and $\tilde{v}$. In this event put

$$
u * v=\tilde{u}^{-1} \circ \tilde{v} .
$$

The product $*$ is a partial binary operation on $R(A, X)$.

An element $v \in R(A, X), v \neq \varepsilon$, is termed cyclically reduced if $v\left(1_{A}\right)^{-1} \neq$ $v(|v|)$. We say that an element $v \in R(A, X)$ admits a cyclic decomposition if $v=c^{-1} \circ u \circ c$, where $c, u \in R(A, X)$ and $u$ is cyclically reduced. Observe that a cyclic decomposition is unique (whenever it exists). We denote by $C R(A, X)$ the set of all cyclically reduced words in $R(A, X)$ and by $C D R(A, X)$ the set of all words from $R(A, X)$ which admit a cyclic decomposition.

Below we refer to $A$-words as infinite words, usually omitting the $A$ whenever it does not produce any ambiguity.

The following result establishes the connection between infinite words and length functions.

Theorem 2 ([32]). Let $A$ be a discretely ordered abelian group and $X$ be a set. Then any subgroup $G$ of $C D R(A, X)$ has a free Lyndon length function with values in $A$ - the restriction $\left.L\right|_{G}$ on $G$ of the standard length function $L$ on $C D R(A, X)$.

The converse of the theorem above was obtained by I. Chiswell [7].

Theorem 3 ([7]). Let $G$ have a free Lyndon length function $L: G \rightarrow A$, where $A$ is a discretely ordered abelian group. Then there exists a set $X$ and a length preserving embedding $\phi: G \rightarrow C D R(A, X)$, that is, $|\phi(g)|=L(g)$ for any $g \in G$.

In fact, Chiswell proved that there exists a length preserving embedding $\phi: G \rightarrow$ $R(A, X)$, where $\phi(G)$ does not contain elements of order 2. But this is equivalent to $\phi(G) \subset C D R(A, X)$ (see [32]). 
Corollary 1 ([7]). Let $G$ have a free Lyndon length function $L: G \rightarrow A$, where $A$ is an arbitrary ordered abelian group. Then there exists an embedding $\phi: G \rightarrow$ $C D R\left(A^{\prime}, X\right)$, where $A^{\prime}=\mathbb{Z} \oplus A$ is discretely ordered with respect to the right lexicographic order and $X$ is some set such that $|\phi(g)|=(0, L(g))$ for any $g \in G$.

Theorems 2 and 3 , and Corollary 1 show that a group has a free Lyndon length function if and only if it embeds into a set of infinite words and that this embedding preserves the length. Moreover, it is not hard to show that this embedding also preserves regularity of the length function.

Theorem 4 ([19]). Let $G$ have a free regular Lyndon length function $L: G \rightarrow A$, where $A$ is an arbitrary ordered abelian group. Then there exists an embedding $\phi: G \rightarrow R\left(A^{\prime}, X\right)$, where $A^{\prime}$ is a discretely ordered abelian group and $X$ is some set such that the Lyndon length function on $\phi(G)$ induced from $R\left(A^{\prime}, X\right)$ is regular.

\section{Commutation in infinite WORdS}

Let $G$ be a subgroup of $C D R(A, X)$, where $A$ is a discretely ordered abelian group. We fix $G$ for the rest of the section.

Let $I_{A}$ index the set of all convex subgroups of $A$. $I_{A}$ is linearly ordered (see, for example, [6]): $i<j$ if and only if $A_{i}<A_{j}$, and

$$
A=\bigcup_{i \in I_{A}} A_{i} .
$$

We say that $g \in G$ has the height $i \in I_{A}$ and denote $h t(g)=i$ if $|g| \in A_{i}$ and $|g| \notin A_{j}$ for any $j<i$. Observe that this definition depends only on $G$ since the complete chain of convex subgroups of $A$ is unique.

It is easy to see that

$$
h t\left(g_{1} g_{2}\right) \leq \max \left\{h t\left(g_{1}\right), h t\left(g_{2}\right)\right\} .
$$

Hence, if $G=\left\langle g_{1}, \ldots, g_{k}\right\rangle$, then we define

$$
h t(G)=\max \left\{h t\left(g_{1}\right), \ldots, h t\left(g_{k}\right)\right\} .
$$

Using the characteristics of elements of $G$ introduced above we prove several technical results.

Lemma 5. Let $f, h \in G$ be cyclically reduced. If $c\left(f^{m}, h^{n}\right) \geqslant|f|+|h|$ for some $m, n>0$, then $[f, h]=\varepsilon$.

Proof. Suppose $|h| \geqslant|f|$ and $c\left(f^{m}, h^{n}\right) \geqslant|f|+|h|$ for some $m, n>0$. Notice that $c\left(f^{m}, h^{n}\right) \geqslant|f|+|h|$ implies $h t(f)=h t(h)$. Hence, there exists $k \in \mathbb{N}$ such that $|h| \geq k|f|,|h| \leq(k+1)|f|$.

We have $h=f^{k} \circ h_{1},|f|>\left|h_{1}\right|, h_{1} \in G, k \geqslant 1$ and $f=h_{1} \circ f_{1}$. Since $c\left(f^{m}, h^{n}\right) \geqslant|f|+|h|$ one has $\left(f^{k} \circ h_{1}\right) \circ f=f^{k+1} \circ h_{1}$. So, $h_{1} \circ h_{1} \circ f_{1}=h_{1} \circ f_{1} \circ h_{1}$ and $f=h_{1} \circ f_{1}=f_{1} \circ h_{1}$. It follows that $\left[f_{1}, h_{1}\right]=\varepsilon$, hence $\left[h_{1}, f\right]=\varepsilon$, and $[f, h]=\varepsilon$.

Lemma 6. For any two $g_{1}, g_{2} \in G$ if $\left[g_{1}, g_{2}\right]=\varepsilon$ and $g_{1}=c^{-1} \circ h_{1} \circ c, g_{2}=$ $d^{-1} \circ h_{2} \circ d$ are their cyclic decompositions, then $c=d$.

Proof. Without loss of generality we can assume that $c=\varepsilon$. From $\left[g_{1}, g_{2}\right]=\varepsilon$ we get

$$
\left(d^{-1} \circ h_{2}^{-1} \circ d\right) * h_{1} *\left(d^{-1} \circ h_{2} \circ d\right)=h_{1},
$$


where $h_{1} \neq \varepsilon, h_{2} \neq \varepsilon$. Since $h_{1}$ is cyclically reduced, either

$$
\left(d^{-1} \circ h_{2}^{-1} \circ d\right) * h_{1}=\left(d^{-1} \circ h_{2}^{-1} \circ d\right) \circ h_{1}
$$

or

$$
h_{1} *\left(d^{-1} \circ h_{2} \circ d\right)=h_{1} \circ\left(d^{-1} \circ h_{2} \circ d\right) .
$$

Assume the latter (otherwise the argument is similar). Hence, we have

$$
h_{1} \circ\left(d^{-1} \circ h_{2}^{-1} \circ d\right)=\left(d^{-1} \circ h_{2}^{-1} \circ d\right) \circ h_{1},
$$

and unless $d=\varepsilon$ we have $c\left(d^{-1}, h_{1}^{-1}\right)>0$, that is, $h_{1} * d^{-1} \neq h_{1} \circ d^{-1}$ - a contradiction to our assumption. Thus, $d=\varepsilon$.

In particular, it follows that if $g \in G$ is cyclically reduced, then all elements of $C_{G}(g)$ are cyclically reduced as well. In this event we call $C_{G}(g)$ cyclically reduced.

Lemma 7. Let $f, h \in G$ be such that $h$ is cyclically reduced and $h t(f)>h t(h)$. If $h t\left(f^{-1} * h * f\right)<h t(f)$, then for every $n \in \mathbb{N}$ either $f=h^{n} \circ f_{n}$ or $f=h^{-n} \circ f_{n}$ for some $f_{n} \in G$.

Proof. Since $h$ is cyclically reduced, either $f^{-1} * h=f^{-1} \circ h$ or $h * f=h \circ f$. Assume the latter (otherwise the argument is similar).

Assume that the statement holds for some $k \geq 0$, that is, $f=h^{k} \circ f_{k}$. At the same time, $f^{-1} *(h \circ f)=f_{k}^{-1} *\left(h \circ f_{k}\right)$ and $h t\left(f_{k}^{-1} *\left(h \circ f_{k}\right)\right)<h t\left(f_{k}\right)=h t(f)$ implies that $h$ cancels completely in the product $f_{k}^{-1} *\left(h \circ f_{k}\right)$. Hence, $f_{k}$ has $h$ as an initial subword and the statement holds for $k+1$.

Lemma 8. Let $f, h_{1}, h_{2} \in G$ be such that $h t\left(h_{1}\right), h t\left(h_{2}\right)<h t(f)$ and $h t\left(f^{-1} * h_{1} * f\right)$, $h t\left(f^{-1} * h_{2} * f\right)<h t(f)$. Then $\left[h_{1}, h_{2}\right]=\varepsilon$.

Proof. Without loss of generality we can assume $h_{1}$ to be cyclically reduced. Indeed, if $h_{1}=d^{-1} \circ \overline{h_{1}} \circ d$ is the cyclic decomposition of $h_{1}$, then it follows that $f=d^{-1} \circ g$ and

$$
f^{-1} * h_{1} * f=g^{-1} * \overline{h_{1}} * g, f^{-1} * h_{2} * f=g^{-1} *\left(d * h_{2} * d^{-1}\right) * g,
$$

so, one can consider the triple $g, \overline{h_{1}}, d * h_{2} * d^{-1}$ instead of $f, h_{1}, h_{2}$.

Since $h_{1}$ is cyclically reduced it follows that in the product $f^{-1} * h_{1} * f$ either $f^{-1} * h_{1}=f^{-1} \circ h_{1}$ or $h_{1} * f=h_{1} \circ f$. Assume the latter. Now, from $h t\left(f^{-1} * h_{1} * f\right)<$ $h t(f)$ by Lemma 7, $f$ contains any positive power of $h_{1}$ as an initial subword.

At the same time, $h_{2}=c^{-1} \circ \overline{h_{2}} \circ c$, where $\overline{h_{2}}$ is cyclically reduced, and $f=c^{-1} \circ f_{1}$. Thus, $f^{-1} * h_{2} * f=f_{1}^{-1} * \overline{h_{2}} * f_{1}, h t\left(f_{1}^{-1} * \overline{h_{2}} * f_{1}\right)<h t\left(f_{1}\right)$ and by Lemma 7 we can assume that $f_{1}$ contains any positive power of $\overline{h_{2}}$ as an initial segment (for negative power the argument is similar).

Consider several cases.

(1) $h t(c) \leqslant h t\left(h_{1}\right)$.

(a) $h t\left(h_{1}\right)=h t\left(\overline{h_{2}}\right)$.

In this case there exists $n \geq 0$ such that $h_{1}^{n}=c^{-1} \circ \bar{h}_{2}^{\delta} \circ h_{3}$, where $\overline{h_{2}}=h_{3} \circ h_{4}, \delta \geq 0$. Hence, $h t\left(h_{1}\right)=h t\left(h_{4} \circ h_{3}\right)$, both $h_{1}$ and $h_{4} \circ h_{3}$ are cyclically reduced and $c\left(h_{1}^{k},\left(h_{4} \circ h_{3}\right)^{m}\right) \geqslant\left|h_{1}\right|+\left|h_{4} \circ h_{3}\right|$ for some $k, m>0$. By Lemma 5 we have $\left[h_{1}, h_{4} \circ h_{3}\right]=\varepsilon$, that is, 
$C_{G}\left(h_{1}\right)=C_{G}\left(h_{4} \circ h_{3}\right)$ since $G$ is commutative-transitive. It follows that

$$
\begin{gathered}
C_{G}\left(h_{2}\right)=c^{-1} * C_{G}\left(\overline{h_{2}}\right) * c=c^{-1} * C_{G}\left(h_{3} \circ h_{4}\right) * c \\
=c^{-1} * h_{4}^{-1} * C_{G}\left(h_{4} \circ h_{3}\right) * h_{4} * c=c^{-1} * h_{4}^{-1} * C_{G}\left(h_{1}\right) * h_{4} * c .
\end{gathered}
$$

On the other hand, $h_{1}^{n}=c^{-1} \circ h_{3}$, so

$$
\begin{gathered}
C_{G}\left(h_{2}\right)=\left(c^{-1} * h_{4}^{-1} * h_{3}^{-1} * c\right) * C_{G}\left(h_{1}\right) *\left(c^{-1} * h_{3} * h_{4} * c\right) \\
=h_{2}^{-1} * C_{G}\left(h_{1}\right) * h_{2} .
\end{gathered}
$$

That is, $C_{G}\left(h_{1}\right)=C_{G}\left(h_{2}\right)$ and $\left[h_{1}, h_{2}\right]=\varepsilon$.

(b) $h t\left(h_{1}\right)<h t\left(\overline{h_{2}}\right)$.

There exists $n \geq 0$ such that $c^{-1}=h_{1}^{n} \circ h_{3}$, where $h_{1}=h_{3} \circ h_{4}$. Hence, from $h t\left(h_{1}\right)<h t\left(\overline{h_{2}}\right)$ it follows that $\overline{h_{2}}$ contains any positive power of $h_{4} \circ h_{3}$ as an initial subword.

Observe that $f^{-1} * h_{1} * f=f_{1}^{-1} *\left(c * h_{1} \circ c^{-1}\right) * f_{1}=f_{1}^{-1} *\left(c *\left(h_{3} \circ\right.\right.$ $\left.\left.h_{4}\right) * c^{-1}\right) * f_{1}=f_{1}^{-1} *\left(h_{4} \circ h_{3}\right) * f_{1}$ and $h t\left(f_{1}^{-1} *\left(h_{4} \circ h_{3}\right) * f_{1}\right)<h t\left(f_{1}\right)$. From $h t\left(f_{1}^{-1} *\left(h_{4} \circ h_{3}\right) * f_{1}\right)<h t\left(f_{1}\right)$ and $h t\left(f_{1}^{-1} * \overline{h_{2}} * f_{1}\right)<h t\left(f_{1}\right)$ we get $h t\left(f_{1}^{-1} *\left(\left(h_{4} \circ h_{3}\right) \circ \overline{h_{2}}\right) * f_{1}\right)<h t\left(f_{1}\right)$. It follows that $f_{1}$ contains any positive power of $\left(h_{4} \circ h_{3}\right) \circ \overline{h_{2}}$ as an initial subword and $h t\left(\left(h_{4} \circ h_{3}\right) \circ \overline{h_{2}}\right)=h t\left(\overline{h_{2}}\right)$. By Lemma [5 we have $\left[\left(h_{4} \circ h_{3}\right) \circ \overline{h_{2}}, \overline{h_{2}}\right]=\varepsilon$ or, equivalently, $\left[h_{4} \circ h_{3}, \overline{h_{2}}\right]=\varepsilon$. That is, $C_{G}\left(\overline{h_{2}}\right)=C_{G}\left(h_{4} \circ h_{3}\right)$. Now, $h_{4} * C_{G}\left(h_{3} \circ h_{4}\right) * h_{4}^{-1}=C_{G}\left(\overline{h_{2}}\right)$ and $C_{G}\left(h_{1}\right)=h_{4}^{-1} * C_{G}\left(\overline{h_{2}}\right) * h_{4}$. From $c^{-1} \circ h_{4}=h_{1}^{n+1}$ it follows that $h_{4}=c * h_{1}^{n+1}$ and

$$
C_{G}\left(h_{1}\right)=h_{1}^{-(n+1)} * c^{-1} * C_{G}\left(\overline{h_{2}}\right) * c * h_{1}^{(n+1)}=h_{1}^{-(n+1)} * C_{G}\left(h_{2}\right) * h_{1}^{(n+1)} \text {. }
$$

Hence, $C_{G}\left(h_{1}\right)=C_{G}\left(h_{2}\right)$ and $\left[h_{1}, h_{2}\right]=\varepsilon$.

(c) $h t\left(h_{1}\right)>h t\left(\overline{h_{2}}\right)$.

There exists $n \geq 0$ such that $c^{-1}=h .1^{n} \circ h_{3}$, where $h_{1}=h_{3} \circ h_{4}$ and $h_{4} \circ h_{3}$ contains any positive power of $\overline{h_{2}}$ as an initial segment. Similar to (b) we get $\left[h_{4} \circ h_{3}, \overline{h_{2}}\right]=\varepsilon$ and derive $C_{G}\left(h_{1}\right)=C_{G}\left(h_{2}\right)$.

(2) $h t(c)>h t\left(h_{1}\right)$.

It follows that $c^{-1}$ contains any positive power of $h_{1}$ as an initial segment. Consider $f^{-1} *\left(h_{1} \circ h_{2}\right) * f$. We have

$$
\begin{gathered}
f^{-1} *\left(h_{1} \circ h_{2}\right) * f=f^{-1} *\left(h_{1} \circ c^{-1} \circ \overline{h_{2}} \circ c\right) * f \\
=\left(f_{1}^{-1} \circ c\right) *\left(h_{1} \circ c^{-1} \circ \overline{h_{2}} \circ c\right) *\left(c^{-1} \circ f_{1}\right)=\left(f_{1}^{-1} \circ c\right) *\left(h_{1} \circ c^{-1} \circ \overline{h_{2}} \circ f_{1}\right) .
\end{gathered}
$$

Hence, $h_{1} \circ c^{-1}$ cancels completely in $\left(f_{1}^{-1} \circ c\right) *\left(h_{1} \circ c^{-1}\right)$ and $c *\left(h_{1} \circ c^{-1}\right)=$ $h_{3}$ so that $\left|h_{1}\right|=\left|h_{3}\right|$. In particular, $h_{3}$ is cyclically reduced as a cyclic permutation of $h_{1}$. Thus, $\left(f_{1}^{-1} \circ c\right) *\left(h_{1} \circ c^{-1} \circ \overline{h_{2}} \circ f_{1}\right)=f_{1}^{-1} *\left(h_{3} \circ \overline{h_{2}} \circ f_{1}\right)$, and since $h t\left(f_{1}^{-1} *\left(h_{3} \circ \overline{h_{2}}\right) * f_{1}\right)<h t\left(f_{1}\right)$, by Lemma $7 f_{1}$ contains any positive power of $h_{3} \circ \overline{h_{2}}$ as an initial subword. On the other hand, $h t\left(f^{-1} * h_{1} * f\right)<$ $h t(f)$, so $h t\left(\left(f_{1}^{-1} \circ c\right) * h_{1} *\left(c^{-1} \circ f_{1}\right)\right)=h t\left(f_{1}^{-1} *\left(h_{3} \circ f_{1}\right)\right)<h t\left(f_{1}\right)$, and it follows that $f_{1}$ contains any positive power of $h_{3}$ as an initial segment. Using Lemma [5] we get $\left[h_{3}, \overline{h_{2}}\right]=\varepsilon$ and $C_{G}\left(\overline{h_{2}}\right)=C_{G}\left(h_{3}\right)=c * C_{G}\left(h_{1}\right) * c^{-1}$. Hence, $C_{G}\left(h_{2}\right)=c^{-1} * C_{G}\left(\overline{h_{2}}\right) * c=C_{G}\left(h_{1}\right)$ and $\left[h_{1}, h_{2}\right]=\varepsilon$. 
Lemma 9. Let $f, h_{1} \neq \epsilon \in G$ be such that $f$ is cyclically reduced and $h t\left(h_{1}\right)<$ $h t(f)$. If $h t\left(f^{-1} * h_{1} * f\right)<h t(f)$ and $\left[h_{1}, h_{2}\right]=\varepsilon$, where $h t\left(h_{2}\right)<h t(f)$, then $h t\left(f^{-1} * h_{2} * f\right)<h t(f)$.

Proof. Without loss of generality we can assume $h_{1}$ to be cyclically reduced. Indeed, if $h_{1}=a^{-1} \circ \overline{h_{1}} \circ a$ is the cyclic decomposition of $h_{1}$, then by Lemma $6 h_{2}=a^{-1} \circ \overline{h_{2}} \circ a$ is the cyclic decomposition of $h_{2}$ and from $h t\left(f^{-1} *\left(a^{-1} \circ \overline{h_{1}} \circ a\right) * f\right)<h t(f)$ it follows that $f=a^{-1} \circ w_{1}$. Hence, $w_{2}=w_{1} \circ a^{-1}$ is cyclically reduced, and from $w_{2}^{-1} * \overline{h_{1}} * w_{2}=\left(a \circ w_{1}^{-1}\right) * \overline{h_{1}} *\left(w_{1} \circ a^{-1}\right)=a *\left(w_{1}^{-1} * \overline{h_{1}} * w_{1}\right) * a^{-1}$ we get $h t\left(w_{2}^{-1} * \overline{h_{1}} * w_{2}\right)<h t\left(w_{2}\right)$. That is, we can consider the triple $w_{2}, \overline{h_{1}}, \overline{h_{2}}$ instead of $f, h_{1}, h_{2}$.

Since $h_{1}$ is cyclically reduced it follows that in the product $f^{-1} * h_{1} * f$ either $f^{-1} * h_{1}=f^{-1} \circ h_{1}$ or $h_{1} * f=h_{1} \circ f$. Assume the latter. Now, from $h t\left(f^{-1} * h_{1} * f\right)<$ $h t(f)$, by Lemma 7, $f$ contains any positive power of $h_{1}$ as an initial segment.

Since $\left[h_{1}, h_{2}\right]=\varepsilon$, then by Lemma $6 h_{2}$ is cyclically reduced and we have

$$
\left(f^{-1} * h_{1} * f\right) *\left(f^{-1} * h_{2} * f\right)=\left(f^{-1} * h_{2} * f\right) *\left(f^{-1} * h_{1} * f\right) .
$$

Denote $g=f^{-1} * h_{1} * f$ and let $g=c^{-1} \circ \bar{g} \circ c$ be the cyclic decomposition of $g$. Notice that $h t(c)<h t(f)$. Now,

$$
\left(f^{-1} * h_{2}^{-1} * f\right) * g *\left(f^{-1} * h_{2} * f\right)=g,
$$

and conjugating both sides by $c^{-1}$ we get

$$
\left(f_{1}^{-1} * h_{2}^{-1} * f_{1}\right) * \bar{g} *\left(f_{1}^{-1} * h_{2} * f_{1}\right)=\bar{g},
$$

where $f=f_{1} \circ c$.

Suppose the statement of the lemma does not hold, that is, $h t\left(f_{1}^{-1} * h_{2} * f_{1}\right)=$ $h t(f)$. Then $h t\left(f_{1}^{-1} * h_{2}^{-1} * f_{1}\right)=h t(f)$ and $f_{1}^{-1} * h_{2}^{-1} * f_{1}=f_{2}^{-1} \circ h_{3}^{-1} \circ f_{2}$, where $f_{1}=d \circ f_{2}, h t\left(f_{2}\right)=h t(f), h t\left(h_{3}\right)<h t(f)$. Since $\left[f_{1}^{-1} * h_{2}^{-1} * f_{1}, \bar{g}\right]=\left[f_{2}^{-1} \circ h_{3}^{-1} \circ\right.$ $\left.f_{2}, \bar{g}\right]=\varepsilon$, by Lemma 6] we have $f_{2}=\epsilon$. Then $h t\left(f_{1}^{-1} * h_{2} * f_{1}\right)=h t\left(h_{3}\right)<h t(f)$, which contradicts the assumption.

Proposition 1 ([1]). If $G<C D R(A, X)$, then for any $g \in G$, its centralizer $C_{G}(g)$ is a subgroup of $A$. In particular, if $A=\mathbb{Z}^{n}$, then $C_{G}(g)$ is a free abelian group of rank not more than $n$.

\section{Complete $\mathbb{Z}^{n}$-Free Groups}

In this section we fix a finitely generated group $G$ which has a free regular length function with values in $\mathbb{Z}^{n}, n \in \mathbb{Z}$ (with the right lexicographic order). In other words, $G$ is a complete $\mathbb{Z}^{n}$-free group (here, completeness of $G$ means that the length function on $G$ is regular). Due to Theorem 4 we may and will view $G$ as a subgroup of $C D R\left(\mathbb{Z}^{n}, X\right)$ for an appropriate set $X$. Therefore, elements of $G$ are infinite words from $C D R\left(\mathbb{Z}^{n}, X\right)$, multiplication in $G$ is the multiplication $*$ of infinite words, and the regular length function is the standard length $|\cdot|$ of infinite words. That is, $G$ is complete in the sense that it is closed under the operation of taking common initial subwords of its elements.

In the ordered group $\mathbb{Z}^{n}$ with basis $a_{1}, \ldots, a_{n}$ the subgroups $E_{k}=\left\langle a_{1}, \ldots, a_{k}\right\rangle$ are convex, and every non-trivial convex subgroup is equal to $E_{k}$ for some $k$, so

$$
0=E_{0}<E_{1}<\cdots<E_{n}
$$


is the complete chain of convex subgroups of $\mathbb{Z}^{n}$. Recall that the height $h t(g)$ of a word $g \in G$ is equal to $k$ if $|g| \in E_{k}-E_{k-1}$ (see [19]). Since $|g * h| \leq|g|+|h|$ and $\left|g^{-1}\right|=|g|$ one has for any $f, g \in G$ :

(1) $h t(f * g) \leqslant \max \{h t(f), h t(g)\}$,

(2) $h t(g)=h t\left(g^{-1}\right)$.

We will assume that there is an element $g \in G$ with $h t(g)=n$. Otherwise, the length function on $G$ has values in $\mathbb{Z}^{n-1}$, in which case we replace $\mathbb{Z}^{n}$ with $\mathbb{Z}^{n-1}$. For any $k \in[1, n]$

$$
G_{k}=\{g \in G \mid h t(g) \leqslant k\}
$$

is a subgroup of $G$ and

$$
1=G_{0}<G_{1}<\cdots<G_{n}=G .
$$

Observe that if $l: G \rightarrow \Lambda$ is a Lyndon length function with values in some ordered abelian group $\Lambda$ and $\mu: \Lambda \rightarrow \Lambda^{\prime}$ is a homomorphism of ordered abelian groups, then the composition $l^{\prime}=\mu \circ l$ gives a Lyndon length function $l^{\prime}: G \rightarrow \Lambda^{\prime}$. In particular, since $E_{n-1}$ is a convex subgroup of $\mathbb{Z}^{n}$, then the canonical projection $\pi_{n}: \mathbb{Z}^{n} \rightarrow \mathbb{Z}$ such that $\pi_{n}\left(x_{1}, x_{2}, \ldots, x_{n}\right)=x_{n}$ is an ordered homomorphism, so the composition $\pi_{n} \circ|\cdot|$ gives a Lyndon length function $\lambda: G \rightarrow \mathbb{Z}$ such that $\lambda(g)=\pi_{n}(|g|)$. Notice also that if $u=g \circ h$, then $\lambda(u)=\lambda(g)+\lambda(h)$ for any $g, h, u \in G$.

Our goal in this section is to describe the algebraic structure of $G$ in terms of HNN extensions of a very particular type. To this end in Subsection 4.1 we introduce Nielsen-like moves and show that every finite set $Y$ of generators of $G$ can be transformed by a sequence of elementary moves into a set $Z$ with a minimal total $\lambda$-length. Then, in Subsection 4.2. we show that any minimal finite set of generators $Z$ of $G$ gives an HNN-splitting of $G$. Here we use the technique of Stallings' pregroups and their universal groups. Finally, in Subsection 4.3, we use the HNN-splittings obtained earlier to prove the main structure theorem on groups acting freely on $\mathbb{Z}^{n}$-trees.

4.1. Elementary transformations of infinite words. In this section we describe an analog of Nielsen reduction in the group $G$. Since $G$ is complete the Nielsen reduced sets have much stronger non-cancellation properties than usual and the transformations are simpler. On the other hand, since $\mathbb{Z}^{n}$ is non-Archimedean (for $n>1$ ) the reduction process is more cumbersome - it goes in stages along the complete series of convex subgroups in $\mathbb{Z}^{n}$.

For a finite subset $Y$ of $G$ define its $\lambda$-length as

$$
|Y|_{\lambda}=\sum_{g \in Y} \lambda(g)
$$

If $Y$ is a generating set of $G$, then $|Y|_{\lambda}>0$; otherwise $G=G_{n-1}$. It follows that

$$
Y=Y_{+} \cup Y_{0},
$$

where

$$
Y_{+}=\{g \in Y \mid \lambda(g)>0\}, Y_{0}=\{g \in Y \mid \lambda(g)=0\} .
$$

Obviously, $|Y|_{\lambda}=\left|Y_{+}\right|_{\lambda}$ and $\left\langle Y_{0}\right\rangle$ is a finitely generated subgroup of $G_{n-1}$.

Let $Y$ be a finite generating set for $G$. Assuming $Y=Y^{-1}$ we define three types of elementary transformations of $Y$. 
Transformation $\mu$. Let $f, g \in Y_{+}, f \neq g, h \in\left\langle Y_{0}\right\rangle, u=\operatorname{com}(f, h * g)$, and $\lambda(u)>0$. Then $f=u \circ w_{1}, h * g=u \circ w_{2}$ for some $u, w_{1}, w_{2}$ from $G$ (since $G$ is complete). Put

$$
\begin{gathered}
\mu_{f, g, h}(Y)=\left(Y-\left\{f^{ \pm 1}, g^{ \pm 1}\right\}\right) \cup\left\{w_{1}^{ \pm 1}, w_{2}^{ \pm 1}, u^{ \pm 1}\right\} \\
\cup\left\{\left(f^{-1} * h * g\right)^{ \pm 1} \mid \text { if } \lambda\left(f^{-1} * h * g\right)=0\right\}
\end{gathered}
$$

if $f \neq g^{-1}$ and

$$
\mu_{f, g, h}(Y)=\left(Y-\left\{g^{ \pm 1}\right\}\right) \cup\left\{u^{ \pm 1},\left(w_{2} * u\right)^{ \pm 1}\right\}
$$

if $f=g^{-1}$.

Lemma 10. In the notation above

(1) $\langle Y\rangle=\left\langle\mu_{f, g, h}(Y)\right\rangle$,

(2) $\left|\mu_{f, g, h}(Y)\right|_{\lambda}<|Y|_{\lambda}$.

Proof. Observe that $\langle Y\rangle \geq\left\langle\mu_{f, g, h}(Y)\right\rangle$ because $G=\langle Y\rangle$ is complete and $u=$ $\operatorname{com}(f, h * g) \in\langle Y\rangle$.

Suppose $f \neq g^{-1}$. Then $\left\langle\mu_{f, g, h}(Y)\right\rangle \geq\langle Y\rangle$ is obvious and (1) follows. Next,

$$
\lambda(f)+\lambda(g)=2 \lambda(u)+\lambda\left(w_{1}\right)+\lambda\left(w_{2}\right)>\lambda(u)+\lambda\left(w_{1}\right)+\lambda\left(w_{2}\right),
$$

that is, $\left|\mu_{f, g, h}(Y)\right|_{\lambda}<|Y|_{\lambda}$ and (2) follows.

Now, suppose $f=g^{-1}$. Then $\left\langle\mu_{f, g, h}(Y)\right\rangle$ contains $u, w_{2} * u=u^{-1} *(h * g) * u$ and $h$, and hence it contains $g$ as well. Thus, $\left\langle\mu_{f, g, h}(Y)\right\rangle \geq\langle Y\rangle$ and (1) holds. To see (2) let $h=h^{\prime} \circ h^{\prime \prime}, g=\left(h^{\prime \prime}\right)^{-1} \circ g^{\prime}$ so that $h * g=h^{\prime} \circ g^{\prime}$. We have

$$
h^{\prime} \circ g^{\prime}=u \circ w_{2}, g^{-1}=\left(\left(h^{\prime \prime}\right)^{-1} \circ g^{\prime}\right)^{-1}=u \circ w_{1},
$$

where $u=\operatorname{com}\left(g^{-1}, h * g\right)$ and $w_{1}, w_{2} \in G$. That is,

$$
h^{\prime} \circ g^{\prime}=u \circ w_{2},\left(h^{\prime \prime}\right)^{-1} \circ g^{\prime}=w_{1}^{-1} \circ u^{-1} .
$$

First of all notice that $\lambda(g)=\lambda\left(g^{\prime}\right)=\lambda(u)+\lambda\left(w_{1}\right)=\lambda(u)+\lambda\left(w_{2}\right)$, so, since $\lambda(u)>0$, it follows that $\lambda\left(w_{1}\right)=\lambda\left(w_{2}\right)<\lambda\left(g^{\prime}\right)$. Hence, in particular, $\left|w_{2}\right|<\left|g^{\prime}\right|$.

Consider the terminal subwords of length $\left|g^{\prime}\right|$ in both $u \circ w_{2}$ and $w_{1}^{-1} \circ u^{-1}$. Since they are the same, then $|u|<\left|w_{2}\right|$. Indeed, if $|u|>\left|w_{2}\right|$, then $u=w_{1}^{-1} \circ a, u^{-1}=$ $a \circ a^{\prime}$ and $a=a^{-1}$, which is impossible since $a \in G$ and $G \subset C D R\left(\mathbb{Z}^{n}, X\right)$. If $|u|=\left|w_{2}\right|$, then $w_{2}=u^{-1}$ and $h^{\prime} \circ g^{\prime}=u \circ u^{-1}$, which is again impossible. Hence, $w_{2}$ has $u^{-1}$ as a terminal subword, that is, $w_{2}=w \circ u^{-1}$ and

$$
h^{\prime} \circ g^{\prime}=u \circ w_{2}=u \circ w \circ u^{-1}
$$

where

$$
\lambda(g)=\lambda\left(g^{\prime}\right)=2 \lambda(u)+\lambda(w)>\lambda(u)+\lambda(w)=\lambda(u)+\lambda\left(u^{-1} *(h * g) * u\right) .
$$

Thus, $\left|\mu_{f, g, h}(Y)\right|_{\lambda}<|Y|_{\lambda}$.

Transformation $\eta$. Let $f \in Y_{+}$be such that $\lambda(f)>\lambda(\operatorname{com}(f, h * f))>0$ for some $h \in\left\langle Y_{0}\right\rangle$. Then $f=u \circ f_{1}, h * f=u \circ f_{2}, \lambda(u)>0$. Define

$$
\eta_{f, h}(Y)=\left(Y-\left\{f^{ \pm 1}\right\}\right) \cup\left\{f_{1}^{ \pm 1}, u^{ \pm 1},\left(u^{-1} * h * u\right)^{ \pm 1}\right\} .
$$

Notice that $f=\left(h^{-1} * u\right) \circ f_{2}=u \circ f_{1}$, hence $\lambda\left(f_{2}\right)=\lambda\left(f_{1}\right)>0$. On the other hand $f_{2}=\left(u^{-1} * h * u\right) * f_{1}$, and it follows that $\lambda\left(u^{-1} * h * u\right)=0$. 
Lemma 11. In the notation above

(1) $\langle Y\rangle=\left\langle\eta_{f, h}(Y)\right\rangle$,

(2) either $\left|\eta_{f, h}(Y)\right|_{\lambda}<|Y|_{\lambda}$ or $\left|\eta_{f, h}(Y)\right|_{\lambda}=|Y|_{\lambda}$, but then $\left|\eta_{f, h}(Y)_{+}\right|>\left|Y_{+}\right|$.

Proof. Since $G=\langle Y\rangle$ is complete, then $\operatorname{com}(f, h * f) \in\langle Y\rangle$ and we have $\langle Y\rangle \geq$ $\left\langle\eta_{f, h}(Y)\right\rangle$. The inclusion $\left\langle\eta_{f, h}(Y)\right\rangle \geq\langle Y\rangle$ is obvious and (1) holds.

To prove (2), first observe that we always have $\left|\eta_{f, h}(Y)\right|_{\lambda} \leqslant|Y|_{\lambda}$. Finally, $\left|\eta_{f, h}(Y)\right|_{\lambda}=|Y|_{\lambda}$ holds only if $f_{1} \neq u$ and $f_{1}, u \notin Y$, but then $\left|\eta_{f, h}(Y)_{+}\right|>$ $\left|Y_{+}\right|$.

Transformation $\nu$. Let $f \in Y_{+}$not be cyclically reduced. Then $f=c^{-1} \circ \bar{f} \circ c$, where $c \neq 1$ and $\bar{f}$ is cyclically reduced. In this case $c^{-1}=\operatorname{com}\left(f, f^{-1}\right)$, hence (since $G$ is complete) $c, \bar{f} \in G$. Put

$$
\nu_{f}(Y)=\left(Y-\left\{f^{ \pm 1}\right\}\right) \cup\left\{c^{ \pm 1}, \bar{f}^{ \pm 1}\right\} .
$$

Lemma 12. In the notation above

(1) $\langle Y\rangle=\left\langle\nu_{f}(Y)\right\rangle$,

(2) either $\left|\nu_{f}(Y)\right|_{\lambda}<|Y|_{\lambda}$ or $\left|\nu_{f}(Y)\right|_{\lambda}=|Y|_{\lambda}$, but then $\left|\nu_{f}(Y)_{+}\right|=\left|Y_{+}\right|$.

Proof. $\langle Y\rangle \geq\left\langle\nu_{f}(Y)\right\rangle$ follows from the fact that $G=\langle Y\rangle$ is complete, that is, $\operatorname{com}\left(f, f^{-1}\right) \in\langle Y\rangle$. The inclusion $\left\langle\nu_{f}(Y)\right\rangle \geq\langle Y\rangle$ is obvious.

Next, obviously $\left|\nu_{f}(Y)\right|_{\lambda} \leqslant|Y|_{\lambda}$, and $\left|\nu_{f}(Y)\right|_{\lambda}=|Y|_{\lambda}$ only if $\lambda(c)=0$ and $\bar{f} \notin Y$. But it then implies $\left|\nu_{f}(Y)_{+}\right|=\left|Y_{+}\right|$.

We write $Y \rightarrow Y^{\prime}\left(Y \rightarrow^{*} Y^{\prime}\right)$ if $Y^{\prime}$ is obtained from $Y$ by a single (finitely many) elementary transformation, that is, $\rightarrow^{*}$ is the transitive closure of the relation $\rightarrow$. We call a generating set $Y$ of $G$ transformation-reduced if none of the transformations $\mu, \eta, \nu$ can be applied to $Y$. Recall that the binary relation $\rightarrow^{*}$ is called terminating if there is no infinite sequence of finite subsets $Y_{i}, i \in \mathbb{N}$, of $G$ such that $Y_{i} \rightarrow Y_{i+1}$ for every $i \in \mathbb{N}$, i.e., every rewriting system $Y_{1} \rightarrow Y_{2} \rightarrow \cdots$ is finite. We say that $\rightarrow^{*}$ is uniformly terminating if for every finite set $Y$ of $G$ there is a natural number $n_{Y}$ such that every rewriting system starting at $Y$ terminates in at most $n_{Y}$ steps.

Proposition 2. The following hold:

(1) The relation $\rightarrow^{*}$ is uniformly terminating. Moreover, for any finite subset $Y$ of $G$ one has $n_{Y} \leq\left(|Y|_{\lambda}\right)^{3}$. In particular, there exists a finite transformation-reduced $Z \subset G$ which can be obtained from $Y$ in not more than $\left(|Y|_{\lambda}\right)^{3}$ steps.

(2) If $Z$ is a transformation-reduced finite subset of $G$, then:

(a) all elements of $Z_{+}$are cyclically reduced;

(b) if $f, g \in Z_{+}^{ \pm 1}, f \neq g$, then $\lambda(\operatorname{com}(f, h * g))=0$ for any $h \in\left\langle Z_{0}\right\rangle$;

(c) if $f \in Z_{+}^{ \pm 1}$ and $\lambda(\operatorname{com}(f, h * f))>0$ for some $h \in\left\langle Z_{0}\right\rangle$, then $\lambda(\operatorname{com}(f, h * f))=\lambda(f)$.

(3) If $Z$ is a transformation-reduced finite subset of $G$, then one can add to $Z$ finitely many elements $h_{1}, \ldots, h_{m} \in G_{n-1}$ such that $T=Z \cup\left\{h_{1}, \ldots, h_{m}\right\}$ is transformation-reduced and satisfies the following condition:

(d) if $f \in T_{+}^{ \pm 1}$ and $\lambda(\operatorname{com}(f, h * f))>0$ for some $h \in\left\langle T_{0}\right\rangle$, then we get $\lambda(\operatorname{com}(f, h * f))=\lambda(f)$ and $f^{-1} * h * f \in\left\langle T_{0}\right\rangle$. 
Proof. Observe that by Lemma 10 and Lemma 11 the transformations $\mu$ and $\eta$ either decrease $|Y|_{\lambda}$ or increase $\left|Y_{+}\right|$, which is bounded from above by $|Y|_{\lambda}$. Hence, in any sequence of transformations $\mu, \eta, \nu$ applied to $Y$, the number of transformations $\mu, \eta$ is bounded by $\left(|Y|_{\lambda}\right)^{2}$. On the other hand, by Lemma 12 the transformation $\nu$ may keep both $\left|Y_{+}\right|$and $|Y|_{\lambda}$ unchanged, but the number of consecutive applications of $\nu$ is bounded by $|Y|_{\lambda}$ because $\nu$ decreases the number of elements in $Y_{+}$which are not cyclically reduced and $\nu$ cannot be applied to the same element twice. Hence, after not more than $\left(|Y|_{\lambda}\right)^{3}$ transformations of $\mu, \eta, \nu$ applied (in any order) to $Y$, one obtains a finite transformation-reduced $Z \subset G$ which satisfies conditions (a), (b) and (c) listed in (2). Indeed, conditions (a), (b) and (c) only mean that no transformation can be applied. Finally, observe that if $f \in Z_{+}^{ \pm 1}$ and $\lambda(\operatorname{com}(f, h * f))>0$ for some $h \in\left\langle Z_{0}\right\rangle$, then $\lambda\left(f^{-1} * h * f\right)=0$ by (c), that is, $f^{-1} * h * f \in G_{n-1}$. Now, from Lemma 8 and Lemma 9, it follows that for every $g \in G_{n-1}$ we have $f^{-1} * h * f \in G_{n-1}$ if and only if $g \in C_{G_{n-1}}(h)$, and in order for $Z$ to satisfy (d), it is enough to add $C_{G_{n-1}}(h)$ and $f^{-1} * C_{G_{n-1}}(h) * f$ to $Z_{0}$. By Proposition 1 both centralizers are finitely generated and it is enough to add only finitely many elements to $Z_{0}$.

Definition 2. A finite set $Y$ of $G$ is called reduced if it satisfies conditions (a) (d) from Proposition 2 ,

4.2. Minimal sets of generators and pregroups. Let $Z$ be a finite reduced generating set of $G$. Put

$$
P_{Z}=\left\{g * f * h \mid f \in Z_{+}^{ \pm 1}, g, h \in\left\langle Z_{0}\right\rangle\right\} \cup\left\langle Z_{0}\right\rangle .
$$

Multiplication $*$ induces a partial multiplication (which we again denote by $*$ ) on $P_{Z}$ so that for $p, q \in P_{Z}$ the product $p * q$ is defined in $P_{Z}$ if and only if $p * q \in P_{Z}$. Notice that $P_{Z}$ is closed under inversion.

Lemma 13. Let $x=h_{1}(x) * f_{x} * h_{2}(x), y=h_{1}(y) * f_{y} * h_{2}(y) \in P_{Z}$, where $h_{i}(x), h_{i}(y) \in\left\langle Z_{0}\right\rangle, i=1,2$, and $f_{x}, f_{y} \in Z_{+}^{ \pm 1}$. Then $x * y \in P_{Z}$ if and only if $f_{x}=f_{y}^{-1}$ and $f_{x} *\left(h_{2}(x) * h_{1}(y)\right) * f_{x}^{-1} \in\left\langle Z_{0}\right\rangle$.

Proof. If $f_{x} *\left(h_{2}(x) * h_{1}(y)\right) * f_{y} \in\left\langle Z_{0}\right\rangle$, then since $Z$ is reduced we have $f_{x}=f_{y}^{-1}$ and obviously $x * y \in\left\langle Z_{0}\right\rangle \subset P_{Z}$.

Now, assume $x * y \in P_{Z}$. It follows that

$$
x * y=\left(h_{1}(x) * f_{x} * h_{2}(x)\right) *\left(h_{1}(y) * f_{y} * h_{2}(y)\right)=h_{1}(z) * f_{z} * h_{2}(z) \in P_{Z},
$$

or, in other words,

$$
\left(h_{1}(x) * f_{x} * h_{2}(x)\right) *\left(h_{1}(y) * f_{y} * h_{2}(y)\right) *\left(h_{2}(z)^{-1} * f_{z}^{-1} * h_{1}(z)^{-1}\right)=\varepsilon .
$$

Thus, either we have

$$
\lambda\left(\operatorname{com}\left(f_{x}^{-1},\left(h_{2}(x) * h_{1}(y)\right) * f_{y}\right)\right)>0
$$

or

$$
\lambda\left(\operatorname{com}\left(f_{y}^{-1},\left(h_{2}(y) * h_{2}(z)^{-1}\right) * f_{z}^{-1}\right)\right)>0 .
$$

Suppose the former is true (the latter case is considered similarly). Since $Z$ is reduced it follows that $f_{x}^{-1}=f_{y}$ and $f_{x} *\left(h_{2}(x) * h_{1}(y)\right) * f_{x}^{-1} \in\left\langle Z_{0}\right\rangle$.

Now we are ready to prove the main technical result of this section. 
Theorem 5. Let $G$ be a finitely generated group with a free length function in $\mathbb{Z}^{n}$. Then:

(1) $P_{Z}$ forms a pregroup with respect to the multiplication $*$ and inversion;

(2) the inclusion $P_{Z} \rightarrow G$ extends to the group isomorphism $U\left(P_{Z}\right) \rightarrow G$, where $U(P)$ is the universal group of $P_{Z}$;

(3) if $\left(g_{1}, \ldots, g_{k}\right)$ is a reduced $P_{Z}$-sequence for an element $g \in G$, then

$$
\lambda(g)=\sum_{i=1}^{k} \lambda\left(g_{i}\right) .
$$

Proof. Observe that $P_{Z}=P_{Z}^{-1} \subset G$ generates $G$ and every $g \in G$ corresponds to a finite reduced $P_{Z}$-sequence

$$
\left(u_{1}, u_{2}, \ldots, u_{k}\right)
$$

where $u_{i} \in P_{Z}, i \in[1, k], u_{i} * u_{i+1} \notin P_{Z}, i \in[1, k-1]$, and $g=u_{1} * u_{2} * \cdots * u_{k}$ in $G$. By Theorem 2 of [38, to prove that $P_{Z}$ is a pregroup and the inclusion $P_{Z} \rightarrow G$ extends to the isomorphism $U\left(P_{Z}\right) \rightarrow G$, it is enough to show that all reduced $P_{Z}$-sequences representing the same element have the same $P_{Z}$-length.

Suppose two reduced $P_{Z \text {-sequences }}$

$$
\left(u_{1}, u_{2}, \ldots, u_{k}\right),\left(v_{1}, v_{2}, \ldots, v_{n}\right)
$$

represent the same element $g \in G$. That is,

$$
\left(u_{1} * \cdots * u_{k}\right) *\left(v_{1} * \cdots * v_{n}\right)^{-1}=\varepsilon .
$$

We use induction on $k+n$ to show that $k=n$. If the $P_{Z}$-sequence

$$
\left(u_{1}, \ldots, u_{k}, v_{n}^{-1}, \ldots, v_{1}^{-1}\right)
$$

is reduced, then

$$
u_{1} * \cdots * u_{k} * v_{n}^{-1} * \cdots * v_{1}^{-1} \neq \varepsilon
$$

because $Z$ is a reduced set. Hence,

$$
\left(u_{1}, \ldots, u_{k}, v_{n}^{-1}, \ldots, v_{1}^{-1}\right)
$$

is not reduced and $u_{k} * v_{n}^{-1} \in P_{Z}$. If $u_{k}=h_{1} * f_{1} * g_{1}, v_{n}=h_{2} * f_{2} * g_{2}$, where $h_{i}, g_{i} \in\left\langle Z_{0}\right\rangle$ and $f_{i} \in Z_{+}^{ \pm 1}, i=1,2$, then by Lemma $13 f_{1}=f_{2}$ and $f_{1} *\left(g_{1} * g_{2}^{-1}\right) *$ $f_{2}^{-1}=c \in\left\langle Z_{0}\right\rangle$. It follows that

$$
\left(u_{1}, u_{2}, \ldots, u_{k-1} *\left(h_{1} * c * h_{2}^{-1}\right)\right),\left(v_{1}, v_{2}, \ldots, v_{n-1}\right)
$$

represent the same element $g * v_{n}^{-1} \in G$ and the sum of their lengths is less than $k+n$, so the result follows by induction. Hence, (1) and (2) follow.

Finally we prove (3).

If $g_{i}=h_{1}\left(g_{i}\right) * f_{g_{i}} * h_{2}\left(g_{i}\right), i \in[1, k]$, then $\lambda\left(g_{i}\right)=\lambda\left(f_{g_{i}}\right)$ because $\lambda\left(h_{1}\left(g_{i}\right)\right)=$ $\lambda\left(h_{2}\left(g_{i}\right)\right)=0$. On the other hand, since $Z$ is reduced and $\left(g_{1}, \ldots, g_{k}\right)$ is a reduced $P_{Z}$-sequence, then $\lambda\left(\operatorname{com}\left(g_{i}^{-1}, g_{i+1}\right)\right)=0$ for $i \in[1, k-1]$. In other words, $\lambda\left(g_{i} * g_{i+1}\right)=\lambda\left(g_{i}\right)+\lambda\left(g_{i+1}\right)$ and the result follows.

Corollary 2. $G_{n-1}=\left\langle Z_{0}\right\rangle$.

Proof. From Theorem 5 it follows that $G=U\left(P_{Z}\right)$ and every element $g$ of $G$ can be represented as a reduced $P_{Z}$-sequence $g=\left(g_{1}, \ldots, g_{k}\right)$, where $g_{i} \in P_{Z}$ and $g_{i} * g_{i+1} \notin P_{Z}$ for any $i \in[1, k-1]$. It follows that $g_{i} \notin\left\langle Z_{0}\right\rangle$ for any $i \in[1, k]$. Hence, if $g \in G_{n-1}$, then $\lambda(g)=0$ and it can only be represented by a reduced $P_{Z}$-sequence of length 1 . Therefore, the statement follows from Theorem [5). 


\subsection{Algebraic structure of complete $\mathbb{Z}^{n}$-free groups.}

Theorem 6. Let $G$ be a finitely generated group with a regular free Lyndon length function in $\mathbb{Z}^{n}$ and let $Z$ be a reduced generating set for $G$. Then $G$ has the following presentation:

$$
G=\left\langle H, Y \mid t_{i}^{-1} C_{H}\left(u_{t_{i}}\right) t_{i}=C_{H}\left(v_{t_{i}}\right), t_{i} \in Y^{ \pm 1}\right\rangle,
$$

where $Y=Z_{+}$is finite, $H=G_{n-1}=\left\langle Z_{0}\right\rangle$ is finitely generated and $C_{H}\left(u_{t_{i}}\right) C_{H}\left(v_{t_{i}}\right)$ are either trivial or finitely generated free abelian subgroups of $H$. Moreover, $H$ has a regular free Lyndon length function in $\mathbb{Z}^{n-1}$.

Proof. From Theorem 5 it follows that $G=U\left(P_{Z}\right)$, where

$$
P_{Z}=\left\{g * f * h \mid f \in Z_{+}^{ \pm 1}, g, h \in\left\langle Z_{0}\right\rangle\right\} \cup\left\langle Z_{0}\right\rangle .
$$

It follows that every element $g$ of $G$ can be represented as a reduced $P_{Z}$-sequence $g=\left(g_{1}, \ldots, g_{k}\right)$, where $g_{i} \in P_{Z}-\left\langle Z_{0}\right\rangle$ for any $i \in[1, k]$ and $g_{i} * g_{i+1} \notin P_{Z}$ for any $i \in[1, k-1]$ if $k>1$ (if $k=1$, then $g_{1}$ may be in $\left\langle Z_{0}\right\rangle$ ). In fact (see [37]), we have

$$
\left.G=U(G)=\left\langle P_{Z}\right| x y=z,\left(x, y, z \in P_{Z} \text { and } x * y=z\right)\right\rangle .
$$

Denote $H=\left\langle Z_{0}\right\rangle$ and $Y=Z_{+}$. By Corollary 2 we have $H=G_{n-1}$.

At first observe that $P_{Z}$ is infinite, but for each $p \in P_{Z}$ either $p \in H$ or $p=$ $h_{1}(p) * f_{p} * h_{2}(p)$, where $f_{p} \in Y^{ \pm 1}$ and $h_{i}(p) \in H, i=1,2$. Hence, every $p \in P_{Z}$ can be rewritten in terms of $Y^{ \pm}$and finitely many generators of $H$. On the other hand, if $x, y, z \in P_{Z}$ and $x * y=z$, then one of the three of them is in $H$, and without loss of generality we can assume $z \in H$. Hence, either $x, y$ are in $H$ too, or $x, y \notin H$, and assuming $x=h_{1}(x) * f_{x} * h_{2}(x), y=h_{1}(y) * f_{y} * h_{2}(y)$, where $h_{i}(x), h_{i}(y) \in H, i=1,2, f_{x}, f_{y} \in Y^{ \pm 1}$ by Lemma [13, we get $f_{x}=f_{y}^{-1}$ and $f_{x} *\left(h_{2}(x) * h_{1}(y)\right) * f_{x}^{-1} \in H$. Hence, every relator $x y=z$, where $x, y, z \in P_{Z}$, and $x * y=z$ can be rewritten as

$$
f_{x} * u_{x, y} * f_{x}^{-1}=v_{x, y, z}
$$

where $f_{x} \in Y^{ \pm 1}$ and $u_{x, y}, v_{x, y, z} \in H$. By Lemma 9, for each $q \in Y^{ \pm 1}$ there exists $u_{q} \in H$ such that for each $u \in H$ we have $q * u * q^{-1} \in H$ if and only if $u \in C_{H}\left(u_{q}\right)$. Since $Z$ is reduced it follows that for each $q \in Y^{ \pm 1}$ both $C_{H}\left(u_{q}\right)$ and $q * C_{H}\left(u_{q}\right) * q^{-1}$ are in $H$, and also note that $q * C_{H}\left(u_{q}\right) * q^{-1}$ is a centralizer of some element in $H$. Hence, every

$$
f_{x} * u_{x, y} * f_{x}^{-1}=v_{x, y, z}
$$

is a consequence of

$$
f_{x} * C_{H}\left(u_{x}\right) * f_{x}^{-1}=C_{H}\left(v_{x}\right),
$$

where $u_{x}, v_{x}$ depend only on $f_{x}$. Thus,

$$
G=\left\langle Y, H \mid t_{i}^{-1} C_{H}\left(u_{t_{i}}\right) t_{i}=C_{H}\left(v_{t_{i}}\right), t_{i} \in Y^{ \pm 1}\right\rangle,
$$

where $Y$ is finite, $H$ is finitely generated and $C_{H}\left(u_{y}\right), C_{H}\left(v_{y}\right)$ are finitely generated abelian (see Proposition 1).

Finally, we have to show that $H$ has a regular free Lyndon length function in $\mathbb{Z}^{n-1}$. Indeed, since $H=G_{n-1}<G$, the free Lyndon length function with values in $\mathbb{Z}^{n-1}$ is automatically induced on $H$. We just have to check if it is regular. 
Take $g, h \in H$ and consider $\operatorname{com}(g, h)$. Since the length function on $G$ is regular, $\operatorname{com}(g, h) \in G=U\left(P_{Z}\right)$ and $\operatorname{com}(g, h)$ can be represented by the reduced $P_{Z^{-}}$ sequence $\left(g_{1}, \ldots, g_{k}\right)$. By Theorem 5 (3) it follows that

$$
\lambda(\operatorname{com}(g, h))=\sum_{i=1}^{k} \lambda\left(g_{i}\right) .
$$

But if $\lambda(\operatorname{com}(g, h))>0$, then $\lambda(g), \lambda(h)>0$ - a contradiction with the choice of $g$ and $h$. Hence, $\lambda\left(g_{i}\right)=0, i \in[1, k]$, and it follows that $k=1$. Thus, $\operatorname{com}(g, h)=$ $g_{1} \in H$. This completes the proof of the theorem.

Theorem 7. Let $G$ be a finitely generated group with a regular free Lyndon length function in $\mathbb{Z}^{n}$. Then $G$ can be represented as a union of a finite series of groups

$$
G_{1}<G_{2}<\cdots<G_{n}=G,
$$

where $G_{1}$ is a free group of finite rank, and

$$
G_{i+1}=\left\langle G_{i}, s_{i, 1}, \ldots, s_{i, k_{i}} \mid s_{i, j}^{-1} C_{i, j} s_{i, j}=\phi_{i, j}\left(C_{i, j}\right)\right\rangle \text {, }
$$

where for each $j \in\left[1, k_{i}\right], C_{i, j}$ and $\phi_{i, j}\left(C_{i, j}\right)$ are cyclically reduced centralizers of $G_{i}, \phi_{i, j}$ is an isomorphism, and the following conditions are satisfied:

(1) $C_{i, j}=\left\langle c_{1}^{(i, j)}, \ldots, c_{m_{i, j}}^{(i, j)}\right\rangle, \phi_{i, j}\left(C_{i, j}\right)=\left\langle d_{1}^{(i, j)}, \ldots, d_{m_{i, j}}^{(i, j)}\right\rangle$, where $\phi_{i, j}\left(c_{k}^{(i, j)}\right)=$ $d_{k}^{(i, j)}, k \in\left[1, m_{i, j}\right]$ and

$$
\begin{gathered}
h t\left(c_{k}^{(i, j)}\right)=h t\left(d_{k}^{(i, j)}\right)<h t\left(d_{k+1}^{(i, j)}\right)=h t\left(c_{k+1}^{(i, j)}\right), k \in\left[1, m_{i, j}-1\right], \\
h t\left(s_{i, j}\right)>h t\left(c_{k}^{(i, j)}\right),
\end{gathered}
$$

(2) $\left|\phi_{i, j}(w)\right|=|w|$ for any $w \in C_{i, j}$,

(3) $w$ is not conjugate to $\phi_{i, j}(w)^{-1}$ in $G_{i}$ for any non-trivial $w \in C_{i, j}$,

(4) if $A, B \in\left\{C_{i, 1}, \phi_{i, 1}\left(C_{i, 1}\right), \ldots, C_{i, k_{i}}, \phi_{i, k_{i}}\left(C_{i, k_{i}}\right)\right\}$, then either $A=B$ or $A$ and $B$ are not conjugate in $G_{i}$,

(5) $C_{i, j}$ can appear in the list

$$
\left\{C_{i, k}, \phi_{i, k}\left(C_{i, k}\right) \mid k \neq j\right\}
$$

not more than twice.

Proof. The existence of the series

$$
G_{1}<G_{2}<\cdots<G_{n}=G,
$$

where $G_{i+1}, i \in[1, n-1]$, can be obtained from $G_{i}$ by finitely many HNN extensions in which associated subgroups are maximal abelian of finite rank, follows by induction applying Theorem 6. Also, observe that $G_{1}$ has a free length function with values in $\mathbb{Z}$. Hence, by the result of Lyndon [26] it follows that $G_{1}$ is a free group. Moreover, $G_{1}$ is of finite rank by Theorem 6 .

Now, consider $G_{i+1}$. By Theorem 6 we can assume that

$$
G_{i+1}=\left\langle G_{i}, t_{1}, t_{2}, \ldots, t_{p} \mid t_{j}^{-1} C_{G_{i}}\left(u_{t_{j}}\right) t_{j}=C_{G_{i}}\left(v_{t_{j}}\right)\right\rangle
$$

where

(a) all $t_{j}$ are cyclically reduced,

(b) $G_{i}=\langle Y\rangle, h t\left(t_{j}\right)>h t\left(G_{i}\right)$ and

$$
Y \cup\left\{t_{1}, t_{2}, \ldots, t_{p}\right\}
$$

is a reduced generating set for $G_{i+1}$. 
In particular, $Y \cup\left\{t_{1}, t_{2}, \ldots, t_{p}\right\}$ is reduced, that is, it has the properties listed in Proposition 2 .

At first, we can assume that all $C_{G_{i}}\left(u_{t_{j}}\right), C_{G_{i}}\left(v_{t_{j}}\right)$ are cyclically reduced. Indeed, if not, then by Lemma 6 we have $C_{G_{i}}\left(u_{t_{j}}\right)=c^{-1} \circ B \circ c$, where $B$ is cyclically reduced, $c \in G_{i}$ by regularity of the length function on $G_{i}$, and

$$
\left(t_{j}^{-1} * c^{-1}\right) * B *\left(c * t_{j}\right)=C_{G_{i}}\left(v_{t_{j}}\right)
$$

Thus, we can substitute $t_{j}$ by $c * t_{j}, C_{G_{i}}\left(u_{t_{j}}\right)$ by $B$, and the same can be done for $C_{G_{i}}\left(v_{t_{j}}\right)$.

Observe that conjugation by $t_{j}$ induces an isomorphism between $C_{G_{i}}\left(u_{t_{j}}\right)$ and $C_{G_{i}}\left(v_{t_{j}}\right)$, and since we can assume both centralizers to be cyclically reduced, then from

$$
t_{j}^{-1} * C_{G_{i}}\left(u_{t_{j}}\right) * t_{j}=C_{G_{i}}\left(v_{t_{j}}\right)
$$

it follows that for $a \in C_{G_{i}}\left(u_{t_{j}}\right), b \in C_{G_{i}}\left(v_{t_{j}}\right)$ if $t_{j}^{-1} * a * t_{j}=b$, then $|a|=|b|$. In particular, if

$$
C_{G_{i}}\left(u_{t_{j}}\right)=\left\langle c_{1}^{(i, j)}, \ldots, c_{m_{i, j}}^{(i, j)}\right\rangle,
$$

where we can assume $h t\left(c_{k}^{(i, j)}\right)<h t\left(c_{k+1}^{(i, j)}\right)$ for $k \in\left[1, m_{i, j}-1\right]$, then all $d_{k}^{(i, j)}=$ $t_{j}^{-1} * c_{k}^{(i, j)} * t_{j}$ generate $C_{G_{i}}\left(v_{t_{j}}\right)$ and $\left|c_{k}^{(i, j)}\right|=\left|d_{k}^{(i, j)}\right|$. This proves (1) and (2).

Suppose there exist $w_{1} \in C_{G_{i}}\left(u_{t_{j}}\right)$ and $g \in G_{i}$ such that $g^{-1} * w_{1} * g=w_{2}^{-1}$, where $w_{2}=\phi_{i}\left(w_{1}\right) \in C_{G_{i}}\left(v_{t_{j}}\right)$. Observe that either $h t(g) \leq h t\left(w_{1}\right)=h t\left(w_{2}\right)$ (and in this case $w_{1}$ is a cyclic permutation of $w_{2}^{-1}$ ) or $h t(g)>h t\left(w_{1}\right)$. In the latter case, $g$ has any positive power of $w_{1}^{\delta}, \delta \in\{1,-1\}$ as an initial subword and any positive power of $w_{2}^{-\delta}$ as a terminal subword. Without loss of generality we can assume $\delta=1$. Hence, $t_{j} * g^{-1}=t_{j} \circ g^{-1}$ and

$$
\left(t_{j} \circ g^{-1}\right)^{-1} * w_{1} *\left(t_{j} \circ g^{-1}\right)=w_{1}^{-1} .
$$

Consider $h=\operatorname{com}\left(t_{j} \circ g^{-1},\left(t_{j} \circ g^{-1}\right)^{-1}\right)$. Observe that $h \in G_{i}$ and $\left|h^{-1} * w_{1} * h\right|=$ $\left|w_{1}\right|$. Indeed, $w_{1}$ is cyclically reduced, so either $\left(t_{j} \circ g^{-1}\right)^{-1} * w_{1}=\left(t_{j} \circ g^{-1}\right)^{-1} \circ w_{1}$ or $w_{1} *\left(t_{j} \circ g^{-1}\right)=w_{1} \circ\left(t_{j} \circ g^{-1}\right)$. Assuming the latter (the other case is similar) we have

$$
\left(t_{j} \circ g^{-1}\right)^{-1} * w_{1} *\left(t_{j} \circ g^{-1}\right)=\left(t_{j} \circ g^{-1}\right)^{-1} *\left(w_{1} \circ\left(t_{j} \circ g^{-1}\right)\right),
$$

and from $\left|\left(t_{j} \circ g^{-1}\right)^{-1} * w_{1} *\left(t_{j} \circ g^{-1}\right)\right|=\left|w_{1}\right|$ it follows that $\left(t_{j} \circ g^{-1}\right)^{-1}$ cancels completely in the product $\left(t_{j} \circ g^{-1}\right)^{-1} * w_{1} *\left(t_{j} \circ g^{-1}\right)$. Eventually, since $h$ is an initial subword of $t_{j} \circ g^{-1}$, it follows that $h^{-1}$ cancels completely in the product $h^{-1} *\left(w_{1} \circ h\right)$, so $\left|h^{-1} * w_{1} * h\right|=\left|w_{1}\right|$. Thus, if $w_{3}=h^{-1} * w_{1} * h$, then $h$ ends with any positive power of $w_{3}$. We have $t_{j} \circ g^{-1}=h \circ f \circ h^{-1}$, where $f$ is cyclically reduced. But at the same time we have $f^{-1} * w_{3} * f=w_{3}^{-1}$, and this produces a contradiction. Indeed, if $h t(f) \leq h t\left(w_{3}\right)$, then $w_{3}$ is a cyclic permutation of $w_{3}^{-1}$, which is impossible. On the other hand, if $h t(f)>h t\left(w_{3}\right)$, then $f$ has any power of $w_{3}^{\alpha}, \alpha \in\{1,-1\}$ as an initial subword and any power of $w_{3}^{-\alpha}$ as a terminal subword - a contradiction with the fact that $f$ is cyclically reduced. This proves (3).

To prove (4), assume that two centralizers from the list

$$
C_{G_{i}}\left(u_{t_{1}}\right), \ldots, C_{G_{i}}\left(u_{t_{p}}\right)
$$

are conjugate in $G_{i}$. Denote $C_{1}=C_{G_{i}}\left(u_{t_{1}}\right), C_{2}=C_{G_{i}}\left(u_{t_{2}}\right)$ and let $C_{1}=h^{-1} * C_{2} * h$ for some $h \in G_{i}$. Hence, in (1) every entry of $C_{1}$ can be substituted by $h^{-1} * C_{2} * h$ and some of the elements $t_{1}, t_{2}, \ldots, t_{p}$ can be changed accordingly. 
Finally, assume that there exists $t_{k} \neq t_{j}$ such that $t_{k}^{-1} * C_{G_{i}}\left(u_{t_{j}}\right) * t_{k} \leq G_{i}$. Suppose $c\left(t_{j}, t_{k}\right)>0$ and denote $z=\operatorname{com}\left(t_{j}, t_{k}\right)$. Observe that $h t(z)>h t\left(C_{G_{i}}\left(u_{t_{j}}\right)\right)$. If $h t(z)<h t\left(t_{j}\right)$, then $z$ conjugates $C_{G_{i}}\left(u_{t_{j}}\right)$ into a cyclically reduced centralizer $A$ of $G_{i}$ and $z$ has any positive power of some $a \in A, h t(a)=h t(A)$ as a terminal subword. But then $h t\left(z^{-1} * t_{j}\right)=h t\left(z^{-1} * t_{k}\right)=h t\left(t_{j}\right)$, and since both $z^{-1} * t_{j}$ and $z^{-1} * t_{k}$ conjugate $A$ into a cyclically reduced centralizer of $G_{i}$, it follows that $z^{-1} * t_{j}$ and $z^{-1} * t_{k}$ have $a^{ \pm 1}$ as an initial subword. If $z^{-1} * t_{j}$ has $a$ as an initial subword and $z^{-1} * t_{k}$ has $a^{-1}$ as an initial subword, then $z *\left(z^{-1} * t_{k}\right) \neq z \circ\left(z^{-1} * t_{k}\right)$, and we have a contradiction. If both $z^{-1} * t_{j}$ and $z^{-1} * t_{k}$ have $a$ as an initial subword, then $z$ cannot be $\operatorname{com}\left(t_{j}, t_{k}\right)$ and again we have a contradiction. Thus, $h t(z)=h t\left(t_{j}\right)$, but it is possible only if $t_{j}=t_{k}$ since $Y \cup\left\{t_{1}, t_{2}, \ldots, t_{p}\right\}$ is a minimal generating set, and again we have a contradiction with our choice of $t_{k}$. It follows that $c\left(t_{j}, t_{k}\right)=0$, and if $t_{j}$ begins with $c \in C_{G_{i}}\left(u_{t_{j}}\right)$, then $t_{k}$ begins with $c^{-1}$. It also follows that there can be only one $t_{k} \neq t_{j}$ such that $t_{k}^{-1} * C_{G_{i}}\left(u_{t_{j}}\right) * t_{k} \leq G_{i}$.

This completes the proof of the theorem.

\section{Regular Free aCtions of HNN EXtensions}

Let $H$ be a finitely generated group with a regular free length function with values in $\mathbb{Z}^{n}$. Observe that $\mathbb{Z}^{n} \simeq \bigoplus_{i=0}^{n-1}\left\langle t^{i}\right\rangle$, which is a subgroup of $\mathbb{Z}[t]$, so we can always assume that $H$ has a regular free length function with values in $\mathbb{Z}[t]$. On the other hand, observe that if a group has a free length function with values in $\mathbb{Z}[t]$ and this group is finitely generated, then there exists $n \in \mathbb{N}$ such that the length function takes values in $\mathbb{Z}^{n}$.

The main goal of this section is to prove the following result.

Theorem 8. Let $H$ be a finitely generated group with a regular free length function in $\mathbb{Z}[t]$. Let $A$ and $B$ be centralizers in $H$ whose elements are cyclically reduced and such that there exists an isomorphism $\phi: A \rightarrow B$ with the following properties:

(1) a is not conjugate to $\phi(a)^{-1}$ in $H$ for any $a \in A$,

(2) $|\phi(a)|=|a|$ for any $a \in A$.

Then the group

$$
G=\left\langle H, z \mid z^{-1} A z=B\right\rangle
$$

has a regular free length function in $\mathbb{Z}[t]$ which extends the length function on $H$.

5.1. Cyclically reduced centralizers and attached elements. From Theorem 7. $H$ is a union of the chain

$$
F(X)=H_{1}<H_{2}<\cdots<H_{n}=H,
$$

where

$$
H_{i+1}=\left\langle H_{i}, s_{i, 1}, \ldots, s_{i, k_{i}} \mid s_{i, j}^{-1} C_{i, j} s_{i, j}=D_{i, j}\right\rangle,
$$

$C_{i, j}, D_{i, j}$ are maximal abelian subgroups of $H_{i}$, and $h t\left(s_{i, j}\right)>h t\left(H_{i}\right)$ for any $i \in[1, n-1], j \in\left[1, k_{i}\right]$.

Let $K$ be a cyclically reduced centralizer in $H$. It is easy to see that either $h t(K)=h t(H)=n$ or $h t(K)<h t(H)$ and $K$ is a centralizer from $H_{n-1}$.

For a cyclically reduced centralizer $K$ of $H$ we define

$$
\mathcal{C}(K)=\left\{C_{i, j}, D_{i, j}\right\} \cap\{\text { cyclically reduced centralizers conjugate to } K \text { in } H\} \text {. }
$$


Lemma 14. Let $K$ be a cyclically reduced centralizer of $H$, and let $\mathcal{C}(K)$ be empty. Let a be a generator of $K$ of maximal height. Then there is no element in $H$ which has any positive power of $a^{ \pm 1}$ as an initial subword.

Proof. If $h t(K)=h t(H)$, then the statement is obvious. Suppose $h t(K)<h t(H)$ and there exists $g \in H$ which starts with any positive power of $a^{ \pm 1}$. Consider $w=\operatorname{com}(g, a * g)$ and observe that we have $h t\left(w^{-1} * K * w\right)=h t(K)$. Without loss of generality we assume $h t(w)=n$. Since $w \in H_{n}-H_{n-1}$, there exists the following Britton-reduced representation of $w$,

$$
w=h_{1} * t_{1}^{\epsilon_{1}} * h_{2} * \cdots * t_{k}^{\epsilon_{k}} * h_{k+1},
$$

where $t_{i} \in\left\{s_{n-1,1}, \ldots, s_{n-1, k_{n-1}}\right\}, i \in[1, k], h_{j} \in H_{n-1}, j \in[1, k+1]$. We can assume $\epsilon_{1}=1$.

Denote $C=h_{1}^{-1} * K * h_{1}$, so $h t\left(t_{1}^{-1} * C * t_{1}\right)=h t(C)$. At the same time there exists $A \in\left\{C_{i, j}, D_{i, j}\right\}, i \in[1, n-1], j \in\left[1, k_{i}\right]$ such that $h t\left(t_{1}^{-1} * A * t_{1}\right)=h t(A)$. Thus by Lemma 8 it follows that $C=h_{1}^{-1} * K * h_{1}=A$. But this implies that $\mathcal{C}(K)$ is not empty, a contradiction.

If $\mathcal{C}(K) \neq \emptyset$, then for $C \in \mathcal{C}(K)$ we call $w$ from the list $s_{i, j}, i \in[1, n-1], j \in$ $\left[1, k_{i}\right]$, attached to $C$ if $h t(w)>h t(C)$ and $h t\left(w^{-1} * C * w\right)=h t(C)$. Observe that by Theorem 7, $C$ can have at most two attached elements of the same height, and if $w_{1}, w_{2}, w_{1} \neq w_{2}$ are attached to $C$ and $h t\left(w_{1}\right)=h t\left(w_{2}\right)$, then $w_{1}^{-1} * w_{2}=w_{1}^{-1} \circ w_{2}$.

Below we are going to distinguish attached elements in the following way. Suppose $C \in \mathcal{C}(K)$, and let $w$ be an element attached to $C$. If $c$ is a generator of $C$ of maximal height, then we say that $w$ is left-attached to $C$ with respect to $c$ if $c^{-1} * w=c^{-1} \circ w$ and we say that $w$ is right-attached to $C$ with respect to $c$ if $c * w=c \circ w$.

Lemma 15. Let $K$ be a cyclically reduced centralizer of $H$, and let $C \in \mathcal{C}(K)$. Let $c$ be a generator of $C$ of maximal height. If there exists a right(left)-attached to $C$ with respect to $c$ element, then there exists $D \in \mathcal{C}(K)$ and its generator $d$ of maximal height such that $c$ is conjugate to $d$ in $H$ and $D$ does not have right(left)-attached with respect to d elements.

Proof. Assume that there exists an element $t_{1}$ which is right-attached to $C$ with respect to $c$ (the case of a left-attached element is symmetric). Denote $D_{1}=t_{1}^{-1} *$ $C * t_{1} \in \mathcal{C}(K)$. Observe that $t_{1}^{-1} *\left(c \circ t_{1}\right)=d_{1} \in D_{1}$ and $h t\left(d_{1}\right)=h t\left(D_{1}\right)=h t(C)$. Also, it follows that $d_{1}$ is not a proper power because otherwise $c$ is a proper power and this is not possible. Hence, $d_{1}$ is a primitive element of $D_{1}$, so it is a generator of $D_{1}$ of maximal height. Now, observe that $t_{1}^{-1}$ is a left-attached to $D_{1}$ with respect to $d_{1}$ element. If other attached to $D_{1}$ elements are all left-attached with respect to $d_{1}$, then we are done. Otherwise there exists a right-attached to $D_{1}$ with respect to $d_{1}$ element $t_{2}$. Observe that $t_{1} * t_{2}=t_{1} \circ t_{2}$. Denote $D_{2}=t_{2}^{-1} *\left(D_{1} \circ t_{2}\right) \in \mathcal{C}(K)$ and repeat the argument above.

Since $\mathcal{C}(K)$ is finite. after a finite number of steps either we find $D_{p} \in \mathcal{C}(K), D_{p} \neq$ $C$ and its generator $d_{p}$ of maximal height such that $D_{p}$ has only left-attached elements with respect to $d_{p}$ or we get $D_{p}=D_{p+m}$. Let us consider the latter case. Without loss of generality we can assume $p=1$. Thus, if we denote $w=$ $t_{1} \circ t_{2} \circ \cdots \circ t_{m}$, then $w^{-1} * C * w=C$, that is, $w \in C$. But this gives us a contradiction because $h t(w)>h t(C)$. 
Lemma 16. Let $K$ be a cyclically reduced centralizer of $H$, and let $C \in \mathcal{C}(K)$. Let $c$ be a generator of $C$ of maximal height. If there exists no right(left)-attached to $C$ with respect to $c$ element, then there is no element $g \in H$ which has any positive power of $c$ as an initial(terminal) subword.

Proof. Observe that it is enough to consider only the case when there exists no right-attached to $C$ with respect to $c$ element (the other case is symmetric).

The proof follows from the following claim.

Claim. If there exists $g \in H$ which starts with any positive power of $c$, then there exists $f \in H$ with the same property and such that $h t(f)<h t(g)$.

Suppose there exists $g \in H$ which starts with any positive power of $c$. Without loss of generality we assume $h t(g)=n$. Consider $w=\operatorname{com}(g, \operatorname{cog} g)$. If $h t(w)<h t(g)$, then we are done. So assume $w \in H_{n}-H_{n-1}$.

Observe that we have $h t\left(w^{-1} * C * w\right)=h t(C)$, and without loss of generality we can assume $\lambda(w)$ to be minimal possible (recall that $|w| \in \mathbb{Z}^{n} \subset \mathbb{Z}[t]$ and $\lambda(w)$ is the $n$-th coordinate of $|w|$ in $\left.\mathbb{Z}^{n}\right)$. Since $w \in H_{n}-H_{n-1}$, there exists the following Britton-reduced representation of $w$ :

$$
w=h_{1} * t_{1}^{\epsilon_{1}} * h_{2} * \cdots * t_{k}^{\epsilon_{k}} * h_{k+1},
$$

where $t_{i} \in\left\{s_{n-1,1}, \ldots, s_{n-1, k_{n-1}}\right\}, i \in[1, k], h_{j} \in H_{n-1}, j \in[1, k+1]$. Since this representation is Britton-reduced it follows that

$$
\lambda(w)=\sum_{i=1}^{k} \lambda\left(t_{i}\right)
$$

because $\lambda\left(t_{i}\right)>0, i \in[1, k]$, while $\lambda\left(h_{i}\right)=0, i \in[1, k+1]$. We can assume $\epsilon_{1}=1$. Then $h t\left(\left(h_{1} * t_{1} * h_{2}\right)^{-1} * C *\left(h_{1} * t_{1} * h_{2}\right)\right)=h t(C)$ and $\lambda\left(h_{1} * t_{1} * h_{2}\right) \leq \lambda(w)$. From our assumption it follows that $\lambda\left(h_{1} * t_{1} * h_{2}\right)=\lambda(w)$ and $w=h_{1} * t_{1} * h_{2}$.

Denote $D=h_{1}^{-1} * C * h_{1}$, so $h t\left(t_{1}^{-1} * D * t_{1}\right)=h t(D)$. At the same time there exists $A \in\left\{C_{i, j}, D_{i, j}\right\}, i \in[1, n-1], j \in\left[1, k_{i}\right]$, such that $h t\left(t_{1}^{-1} * A * t_{1}\right)=h t(A)$. Thus by Lemma 8 it follows that $D=h_{1}^{-1} * C * h_{1}=A$ and in particular $h t(C)=h t(A)$.

If $h t\left(h_{1}\right) \leq h t(C)$, then from Theorem 7 it follows that $A=C$ and $h_{1} \in C$. Hence, $t_{1}$ has any positive power of $c$ as an initial subword, and it follows that $t_{1}$ is right-attached to $C$ with respect to $c$, a contradiction. Thus, we have $h t(C)<$ $h t\left(h_{1}\right)<h t(w)=h t(g)$, and the claim is proved.

Now, the existence of an element $g \in H$ starting with any positive power of $a$ implies the existence of such an element $f$ such that $h t(f)=h t(K)$, which is a contradiction.

5.2. Connecting elements. We call a pair of elements $u, v \in C D R(\mathbb{Z}[t], X)$ an admissible pair if

(1) $u, v$ are cyclically reduced,

(2) $u, v$ are not proper powers,

(3) $|u|=|v|$,

(4) $u$ is not conjugate to $v^{-1}$ (in particular, $u \neq v^{-1}$ ).

For an admissible pair $\{u, v\}$ we define an infinite word $s_{u, v} \in R(\mathbb{Z}[t], X)$, which we call the connecting element for the pair $\{u, v\}$, in the following way:

$$
s_{u, v}(\beta)= \begin{cases}u(\alpha) & \text { if } \beta=(k|u|+\alpha, 0), k \geqslant 0,1 \leqslant \alpha \leqslant|u|, \\ v(\alpha) & \text { if } \beta=(-k|v|+\alpha, 1), k \geqslant 1,1 \leqslant \alpha \leqslant|v| .\end{cases}
$$


Since there exists $m>0$ such that $u, v \in C D R\left(\mathbb{Z}^{m}, X\right)-C D R\left(\mathbb{Z}^{m-1}, X\right)$, it is easy to see that $s_{u, v} \in R\left(\mathbb{Z}^{m+1}, X\right)-R\left(\mathbb{Z}^{m}, X\right)$. Also $s_{u, v}^{-1}=s_{v^{-1}, u^{-1}}$ and $u \circ s_{u, v}=s_{u, v} \circ v$ - both follow directly from the definition.

Notice that any two connecting elements $s_{u_{1}, v_{1}}, s_{u_{2}, v_{2}}$ have the same length whenever $u_{1}, v_{1}, u_{2}, v_{2} \in C D R\left(\mathbb{Z}^{m}, X\right)-C D R\left(\mathbb{Z}^{m-1}, X\right)$. In this event we have

$$
\left|s_{u_{1}, v_{1}}\right|=\left|s_{u_{2}, v_{2}}\right|=(0, \ldots, 0,1) \in \mathbb{Z}^{m+1} .
$$

Lemma 17. Let $u, v$ be elements of a group $H \subset C D R(\mathbb{Z}[t], X)$. If the pair $\{u, v\}$ is admissible, then $s_{u, v} \in C D R(\mathbb{Z}[t], X)$.

Proof. Observe that $s_{u, v} \in C D R(\mathbb{Z}[t], X)$ if and only if $\operatorname{com}\left(s_{u, v}, s_{u, v}^{-1}\right)$ exists.

If $c\left(u^{2}, v^{-2}\right) \geq|u|=|v|$, then $c\left(s_{u, v}, s_{u, v}^{-1}\right) \geq|u|=|v|$. Then it follows that $u=v^{-1}$, which is impossible by the assumption. Hence, $c\left(u^{2}, v^{-2}\right)<|u|$, so $c\left(u^{2}, v^{-2}\right)=c\left(u, v^{-1}\right)$ and $\operatorname{com}\left(s_{u, v}, s_{u, v}^{-1}\right)=\operatorname{com}\left(u, v^{-1}\right)$ is defined since $u, v \in$ $H$.

5.3. Main construction. Now, let $A, B$ be cyclically reduced centralizers in $H$ such that there exists an isomorphism $\phi: A \rightarrow B$ satisfying the following conditions:

(1) $a$ is not conjugate to $\phi_{i}(a)^{-1}$ in $H$ for any $a \in A$,

(2) $|\phi(a)|=|a|$ for any $a \in A$.

In particular, it follows that $h t(A)=h t(B)$.

Remark 1. Observe that if $C$ is conjugate to $A$ and $D$ is conjugate to $B$, then

$$
\left\langle H, z \mid z^{-1} A z=B\right\rangle \simeq\left\langle H, z^{\prime} \mid z^{\prime-1} C z^{\prime}=D\right\rangle .
$$

Hence, it is always possible to consider $A$ and $B$ up to taking conjugates.

Let $u$ be a generator of $A$ of maximal height, and let $v=\phi(u) \in B$. Then $v$ is a generator of $B$ of maximal height and $|u|=|v|$. Observe that from the conditions imposed on $\phi$ it follows that the pair $u, v$ is admissible. We fix $u$ and $v$ for the rest of the paper.

Remark 2. Observe that if $\mathcal{C}(A)=\emptyset$, then by Lemma 14, $H$ does not contain an element which has any positive power of $u^{ \pm 1}$ as an initial subword (a similar statement for $B$ and $v$ if $\mathcal{C}(B)=\emptyset$ ). If $\mathcal{C}(A) \neq \emptyset$, then by Lemma 15 we can assume $A$ to have no right-attached elements with respect to $u$. Hence, by Lemma 16. $H$ does not contain an element which has any positive power of $u$ as an initial subword. Similarly, if $\mathcal{C}(B) \neq \emptyset$, then by Lemma [15] we can assume $B$ to have no left-attached elements with respect to $v$. Again, by Lemma 16, $H$ does not contain an element which has any positive power of $v$ as a terminal subword.

Now we are in a position to define $s \in R\left(\mathbb{Z}^{n+1}, X\right)$, which is going to be an infinite word representing $z$ from the presentation (2). Since $\mathbb{Z}[t]$-exponentiation is defined on $C R(\mathbb{Z}[t], X)$ (see [32] for details), for any $f(t) \in \mathbb{Z}[t]$ we can define $v^{f(t)}, u^{f(t)} \in C R(\mathbb{Z}[t], X)$ so that

$$
\left|v^{f(t)}\right|=|v||f(t)|,\left|u^{f(t)}\right|=|u||f(t)|
$$

and

$$
\left[v^{f(t)}, v\right]=\varepsilon,\left[u^{f(t)}, u\right]=\varepsilon .
$$


Thus, if $\alpha=t^{n-h t(A)}$, then $\left|u^{\alpha}\right|=\left|v^{\alpha}\right|=|u||\alpha|$ and $h t\left(u^{\alpha}\right)=h t\left(v^{\alpha}\right)=h t(u)+$ $(n-h t(A))=n$. Hence, we define

$$
s=s_{u^{\alpha}, v^{\alpha}} \in C D R\left(\mathbb{Z}^{n+1}, X\right) .
$$

Observe that $h t(s)=n+1=h t(G)+1$.

Remark 3. It is easy to see that no element of $H$ has $s^{ \pm 1}$ as a subword.

Lemma 18. For any $h \in A$ we have $s^{-1} * h * s=\phi(h) \in B$.

Proof. By the construction above we have $s^{-1} * u * s=v=\phi(u)$, where $u$ is a generator of $A$ of maximal height and $v$ is a corresponding generator of $B$ of maximal height. Now, observe that for every $a \in A$ there exists $r>0$ such that either $a$ or $a^{-1}$ is both an initial and terminal subword of $u^{r}$.

So, let us fix $a \in A$, and without loss of generality we assume that $u^{r}=a \circ$ $u_{1}, u_{1} \in A$. Now,

$$
\phi\left(u_{1}\right) * \phi(a)=\phi(a) * \phi\left(u_{1}\right)=\phi\left(u^{r}\right)=\phi(u)^{r}=v^{r} .
$$

Since $\phi$ preserves length, then

$$
\left|\phi\left(u_{1}\right) * \phi(a)\right|=\left|\phi(a) * \phi\left(u_{1}\right)\right|=\left|v^{r}\right|=\left|u^{r}\right|=|a|+\left|u_{1}\right|=|\phi(a)|+\left|\phi\left(u_{1}\right)\right|,
$$

that is, $\phi(a) * \phi\left(u_{1}\right)=\phi(a) \circ \phi\left(u_{1}\right)=\phi\left(u_{1}\right) \circ \phi(a)$. Hence, the image of an initial subword of $u^{r}$ of length $\alpha$ representing an element of $A$ is both an initial and terminal subword of $v^{r}$ of length $\alpha$.

Finally observe that $s^{-1} * a * s$ is a terminal subword of $s$ of length $|a|$.

Now, our goal is to prove that a pair $H, s$ generates a group in $C D R(\mathbb{Z}[t], X)$.

Lemma 19. For any $g \in H$ there exists $N=N(g)>0$ such that

$$
g * u^{k}=\left(g * u^{N}\right) \circ u^{k-N}, v^{k} * g=v^{k-N} \circ\left(v^{N} * g\right)
$$

for any $k>N$.

Proof. The required result follows immediately from Remark 2 .

Lemma 20. (i) For any $g \in H-A$ there exists $N=N(g)>0$ such that for any $k>N$

$$
u^{-k} * g * u^{k}=u^{-k+N} \circ\left(u^{-N} * g * u^{N}\right) \circ u^{k-N} .
$$

(ii) For any $g \in H$ there exists $N=N(g)>0$ such that for any $k>N$

$$
v^{k} * g * u^{k}=v^{k-N} \circ\left(v^{N} * g * u^{N}\right) \circ u^{k-N} .
$$

(iii) For any $g \in H-B$ there exists $N=N(g)>0$ such that for any $k>N$

$$
v^{k} * g * v^{-k}=v^{k-N} \circ\left(v^{N} * g * v^{-N}\right) \circ u^{-k+N} .
$$

Proof. (i) Observe that by Lemma 19] there exists $M>0$ such that $g * u^{k}=$ $\left(g * u^{M}\right) \circ u^{k-M}$ for all $k>M$. So, without loss of generality we can assume $g * u=g \circ u$. If $h t(g)>h t(u)$, then the required result follows from Remark 2 . Now, assume $h t(g) \leq h t(u)$.

Suppose there is no $N>0$ such that for any $k>N$

$$
u^{-k} * g * u^{k}=u^{-k+N} \circ\left(u^{-N} * g * u^{N}\right) \circ u^{k-N} .
$$


Hence for every $K \in \mathbb{N}$ there are $k, m \in \mathbb{N}$ such that $c\left(u^{k}, g \circ u^{m}\right) \geqslant K|u|$. It follows that $u^{p}=g \circ u_{2}, p>0$, where $u=u_{1} \circ u_{2}$ and $c\left(\left(u_{1} \circ u_{2}\right)^{k},\left(u_{2} \circ u_{1}\right)^{m}\right) \geqslant 2|u|$. Thus, by Lemma 5 it follows that $u_{1} \circ u_{2}=u_{2} \circ u_{1}$ and $[g, u]=\varepsilon$, a contradiction.

(ii) Again, by Lemma 19 there exists $M>0$ such that $g * u^{k}=\left(g * u^{M}\right) \circ u^{k-M}$ for all $k>M$. So, without loss of generality we can assume $g * u=g \circ u$. If $h t(g)>h t(u)$, then the required result follows from Remark 2 Now, assume $h t(g) \leq h t(u)$.

Suppose there is no $N>0$ such that for any $k>N$

$$
v^{k} * g * u^{k}=v^{k-N} \circ\left(v^{N} * g * u^{N}\right) \circ u^{k-N} .
$$

In other words, for every $K \in \mathbb{N}$ there are $k, m \in \mathbb{N}$ such that $c\left(v^{-k}, g \circ u^{m}\right) \geqslant K|u|$. It follows that $v^{-p}=g \circ v_{2}, p>0$, where $v^{-1}=v_{1} \circ v_{2}$ and $u=v_{2} \circ v_{1}=$ $v_{1}^{-1} *\left(v_{1} \circ v_{2}\right) * v_{1}=v_{1}^{-1} * v^{-1} * v_{1}$, a contradiction with the choice of $\phi$.

(iii) The required result follows by the same argument as in (i).

Definition 3. A sequence

$$
p=\left(g_{1}, s^{\epsilon_{1}}, g_{2}, \ldots, g_{k}, s^{\epsilon_{k}}, g_{k+1}\right),
$$

where $g_{j} \in H, \epsilon_{j} \in\{-1,1\}, k \geqslant 1$, is called an $s$-form over $H$.

An $s$-form (3) is reduced if subsequences

$$
\left\{s^{-1}, c, s\right\}, \quad\left\{s, d, s^{-1}\right\},
$$

where $c \in A, d \in B$, do not occur in it.

Denote by $\mathcal{P}(H, s)$ the set of all $s$-forms over $H$. We define a partial function $w: \mathcal{P}(H, s) \rightarrow R(\mathbb{Z}[t], X)$ as follows. If

$$
p=\left(g_{1}, s^{\epsilon_{1}}, g_{2}, \ldots, g_{k}, s^{\epsilon_{k}}, g_{k+1}\right),
$$

then

$$
\left.\left.\left.w(p)=\left(\cdots\left(g_{1} * s^{\epsilon_{1}}\right) * g_{2}\right) * \cdots * g_{k}\right) * s^{\epsilon_{k}}\right) * g_{k+1}\right)
$$

if it is defined.

Lemma 21. Let $p=\left(g_{1}, s^{\epsilon_{1}}, g_{2}, \ldots, g_{k}, s^{\epsilon_{k}}, g_{k+1}\right)$ be an $s$-form over $H$. Then the following hold:

(1) The product $w(p)$ is defined and does not depend on the placement of parentheses.

(2) There exists a reduced s-form $q$ over $H$ such that $w(q)=w(p)$.

(3) If $p$ is reduced, then there exists a unique representation for $w(p)$ of the following type:

$$
\begin{aligned}
w(p)= & \left(g_{1} * u_{1}^{N_{1}}\right) \circ\left(u_{1}^{-N_{1}} * s^{\epsilon_{1}} * v_{1}^{-M_{1}}\right) \circ\left(v_{1}^{M_{1}} * g_{2} * u_{2}^{N_{2}}\right) \\
& \circ \cdots \circ\left(u_{k}^{-N_{k}} * s^{\epsilon_{k}} * v_{k}^{-M_{k}}\right) \circ\left(v_{k}^{M_{k}} * g_{k+1}\right),
\end{aligned}
$$

where $N_{j}, M_{j} \geq 0, u_{j}=u, v_{j}=v$ if $\epsilon_{j}=1$ and $N_{j}, M_{j} \leq 0, u_{j}=v, v_{j}=u$ if $\epsilon_{j}=-1$ for $j \in[1, k]$. Moreover, $g_{1} * u_{1}^{N_{1}}$ does not have $u_{1}^{ \pm 1}$ as a terminal subword, $v_{j-1}^{M_{j-1}} * g_{j} * u_{j}^{N_{j}}$ does not have $u_{j}^{ \pm 1}$ as a terminal subword for every $j \in[2, k]$, and $v_{j-1}^{M_{j-1}} * g_{j} * s_{i}^{\epsilon_{j}}$ does not have $v_{j-1}^{ \pm 1}$ as an initial subword for every $j \in[2, k], v_{k}^{M_{k}} * g_{k+1}$ does not have $v_{k}^{ \pm 1}$ as an initial subword.

(4) $w(p) \in C D R(\mathbb{Z}[t], X)$. 
Proof. Let

be an $s$-form over $H$.

$$
p=\left(g_{1}, s^{\epsilon_{1}}, g_{2}, \ldots, g_{k}, s^{\epsilon_{k}}, g_{k+1}\right)
$$

We show first that (1) implies (2). Suppose that $w(p)$ is defined for every placement of parentheses and all such products are equal. If $p$ is not reduced, then there exists $j \in[2, k]$ such that either $g_{j} \in A, \epsilon_{j-1}=-1, \epsilon_{j}=1$ or $g_{j} \in B, \epsilon_{j-1}=1, \epsilon_{j}=-1$. Without loss of generality we can assume the former. Thus, we have

$$
s^{-1} * g_{j} * s=g_{j}^{\prime} \in B \subseteq H
$$

and we obtain a new $s$-form

$$
p_{1}=\left(g_{1}, s^{\epsilon_{1}}, g_{2}, \ldots, g_{j-1} * g_{j}^{\prime} * g_{j+1}, s^{\epsilon_{j+1}}, \ldots, g_{k}, s^{\epsilon_{k}}, g_{k+1}\right),
$$

which is shorter than $p$ and $w(p)=w\left(p_{1}\right)$. Proceeding this way (or by induction) in a finite number of steps we obtain a reduced $s$-form

$$
q=\left(f_{1}, s^{\delta_{1}}, f_{2}, \ldots, s^{\delta_{l}}, f_{l+1}\right)
$$

such that $w(q)=w(p)$, as required.

Now we show that (1) implies (3). Assume that

$$
p=\left(g_{1}, s^{\epsilon_{1}}, g_{2}, \ldots, g_{k}, s^{\epsilon_{k}}, g_{k+1}\right)
$$

is reduced.

By Lemma 19 and Lemma 20 there exists $r \in \mathbb{N}$ such that for any $\alpha>r$ :

(a) $g_{1} * u^{\alpha}=\left(g_{1} * u^{r}\right) \circ u^{\alpha-r}, g_{1} * v^{-\alpha}=\left(g_{1} * u^{-r}\right) \circ u^{-\alpha+r}$,

(b) $v^{\alpha} * g_{k+1}=v^{\alpha-r} \circ\left(v^{r} * g_{k+1}\right), u^{-\alpha} * g_{k+1}=u^{-\alpha+r} \circ\left(u^{-r} * g_{k+1}\right)$,

(c) $u^{-\alpha} * g_{j} * u^{\alpha}=u^{-(\alpha-r)} \circ\left(u^{-r} * g_{j} * u^{r}\right) \circ u^{\alpha-r}$ for all $j \in[2, k]$ such that $g_{j} \notin A$,

(d) $v^{\alpha} * g_{j} * u^{\alpha}=v^{\alpha-r} \circ\left(v^{r} * g_{j} * u^{r}\right) \circ u^{\alpha-r}$ for all $j \in[2, k]$,

(e) $v^{\alpha} * g_{j} * v^{-\alpha}=v^{\alpha-r} \circ\left(v^{r} * g_{j} * v^{-r}\right) \circ u^{-(\alpha-r)}$ for all $j \in[2, k]$ such that $g_{j} \notin B$.

Since $p$ is reduced, that is, it does not contain either a subsequence $\left\{s^{-1}, g_{j}, s\right\}$, where $g_{j} \in A$, or a subsequence $\left\{s, g_{j}, s^{-1}\right\}$, where $g_{j} \in B$, and $s$ has any power of $u$ as an initial subword and any power of $v$ as a terminal subword, then we have

$$
\begin{gathered}
w(p)=g_{1} * s^{\epsilon_{1}} * g_{2} * \cdots * g_{k} * s^{\epsilon_{k}} * g_{k+1} \\
=\left(g_{1} * u_{1}^{r}\right) \circ\left(u_{1}^{-r} * s^{\epsilon_{1}} * v_{1}^{-r}\right) \circ\left(v_{1}^{r} * g_{2} * u_{2}^{r}\right) \circ \cdots \circ\left(u_{k}^{-r} * s^{\epsilon_{k}} * v_{k}^{-r}\right) \circ\left(v_{k}^{r} * g_{k+1}\right),
\end{gathered}
$$

where $u_{j}=u, v_{j}=v$ if $\epsilon_{j}=1$ and $u_{j}=v^{-1}, v_{j}=u^{-1}$ if $\epsilon_{j}=-1$ for every $j \in[1, k]$.

Now, if $g_{1} * u_{1}^{r}$ has $u_{1}^{\gamma_{1}}, \gamma_{1} \in \mathbb{Z}$ (with $\gamma_{1}$ maximal possible) as a terminal subword, then we denote $N_{1}=r-\gamma_{1}$ and rewrite $w(p)$ as follows:

$$
\begin{aligned}
w(p)= & \left(g_{1} * u_{1}^{N_{1}}\right) \circ\left(u_{1}^{-N_{1}} * s^{\epsilon_{1}} * v_{1}^{-r}\right) \circ\left(v_{1}^{r} * g_{2} * u_{2}^{r}\right) \\
& \circ \cdots \circ\left(u_{k}^{-r} * s^{\epsilon_{k}} * v_{k}^{-r}\right) \circ\left(v_{k}^{r} * g_{k+1}\right) .
\end{aligned}
$$

Now, if $v_{1}^{r} * g_{2} * u_{2}^{r}$ contains $v_{1}^{\delta_{1}}, \delta_{1} \in \mathbb{Z}$ (with $\delta_{1}$ maximal possible) as an initial subword, then we denote $M_{1}=r-\delta_{1}$ and again rewrite $w(p)$ as

$$
\begin{gathered}
w(p)=\left(g_{1} * u_{1}^{N_{1}}\right) \circ\left(u_{1}^{-N_{1}} * s^{\epsilon_{1}} * v_{1}^{-M_{1}}\right) \circ\left(v_{1}^{M_{1}} * g_{2} * u_{2}^{r}\right) \\
\circ \cdots \circ\left(u_{k}^{-r} * s^{\epsilon_{k}} * v_{k}^{-r}\right) \circ\left(v_{k}^{r} * g_{k+1}\right) .
\end{gathered}
$$

In a finite number of steps we obtain the required result. Observe that by the choice of $N_{i}, M_{i}$ the representation of $w(p)$ is unique. 
Now we prove (1) by induction on $k$. If $k=1$, then by Lemma 19 there exists $r \in \mathbb{N}$ such that

$$
\begin{aligned}
g_{1} * u^{\alpha}=\left(g_{1} * u^{r}\right) \circ u^{\alpha-r}, & g_{1} * v^{-\alpha}=\left(g_{1} * v^{-r}\right) \circ v^{-\alpha+r}, \\
v^{\beta} * g_{2}=u^{\beta-r} \circ\left(u^{r} * g_{2}\right), & u^{-\beta} * g_{2}=u^{-\beta+r} \circ\left(v^{-r} * g_{2}\right)
\end{aligned}
$$

for any $\alpha, \beta>r$. Hence,

$$
\begin{aligned}
& \left(g_{1} * s^{\epsilon_{1}}\right) * g_{2}=\left(\left(g_{1} * u_{1}^{r}\right) \circ\left(u_{1}^{-r} * s^{\epsilon_{1}}\right)\right) * g_{2} \\
& =\left(\left(g_{1} * u_{1}^{r}\right) \circ\left(u_{1}^{-r} * s^{\epsilon_{1}} * v_{1}^{-r}\right)\right) \circ\left(v_{1}^{r} * g_{2}\right),
\end{aligned}
$$

where $u_{1}=u, v_{1}=v$ if $\epsilon_{1}=1$ and $u_{1}=v^{-1}, v_{1}=u^{-1}$ if $\epsilon_{1}=-1$. By Theorem 3.4 of [32], the product $\left(g_{1} * s^{\epsilon_{1}}\right) * g_{2}$ does not depend on the placement of the parentheses. So (1) holds for $k=1$.

Now, consider an initial $s$-subsequence of $p$,

$$
p_{1}=\left(g_{1}, s^{\epsilon_{1}}, g_{2}, \ldots, g_{k-1}, s^{\epsilon_{k-1}}, g_{k}\right) .
$$

By induction $w\left(p_{1}\right)$ is defined and does not depend on the placement of parentheses. By the argument above there exists a unique representation of $w\left(p_{1}\right)$,

$$
\begin{gathered}
w\left(p_{1}\right)=\left(g_{1} * u_{1}^{N_{1}}\right) \circ\left(u_{1}^{-N_{1}} * s^{\epsilon_{1}} * v_{1}^{-M_{1}}\right) \circ\left(v_{1}^{M_{1}} * g_{2} * u_{2}^{N_{2}}\right) \\
\circ \cdots \circ\left(u_{k-1}^{-N_{k-1}} * s^{\epsilon_{k-1}} * v_{k-1}^{-M_{k-1}}\right) \circ\left(v_{k-1}^{M_{k-1}} * g_{k}\right),
\end{gathered}
$$

where $N_{j}, M_{j} \geq 0, u_{j}=u, v_{j}=v$ if $\epsilon_{j}=1$, and $N_{j}, M_{j} \leq 0, u_{j}=v, v_{j}=u$ if $\epsilon_{j}=-1$ for $j \in[1, k-1]$. To prove that $p$ satisfies (1) it suffices to show that

$$
w\left(p_{1}\right) *\left(s^{\epsilon_{k}} * g_{k+1}\right)
$$

is defined and does not depend on the placement of parentheses.

Without loss of generality we assume $\epsilon_{k-1}=1, \epsilon_{k}=1$. Other combinations of $\epsilon_{k-1}$ and $\epsilon_{k}$ are considered similarly.

By Lemma 20

$$
\begin{gathered}
\left(\left(u^{-N_{k-1}} * s * v^{-M_{k-1}}\right) \circ\left(v^{M_{k-1}} * g_{k}\right)\right) *\left(s * g_{k+1}\right)=\left(u^{-N_{k-1}} * s * v^{-M_{k-1}-r}\right) \\
\circ\left(v^{M_{k-1}+r} * g_{k} * u^{m_{1}}\right) \circ\left(u^{-m_{1}} * s * v^{-m_{2}}\right) \circ\left(v^{m_{2}} * g_{k+1}\right)
\end{gathered}
$$

for some $m_{1}, m_{2}, r \in \mathbb{N}$. Thus $w\left(p_{1}\right) *\left(s * g_{k+1}\right)$ is defined and does not depend on the placement of the parentheses.

Now we prove (4). By (3) there exists a unique representation of $w(p)$,

$$
\begin{aligned}
w(p)= & \left(g_{1} * u_{1}^{N_{1}}\right) \circ\left(u_{1}^{-N_{1}} * s^{\epsilon_{1}} * v_{1}^{-M_{1}}\right) \circ\left(v_{1}^{M_{1}} * g_{2} * u_{2}^{N_{2}}\right) \\
& \circ \cdots \circ\left(u_{k}^{-N_{k}} * s^{\epsilon_{k}} * v_{k}^{-M_{k}}\right) \circ\left(v_{k}^{M_{k}} * g_{k+1}\right),
\end{aligned}
$$

where $N_{j}, M_{j} \geq 0, u_{j}=u, v_{j}=v$ if $\epsilon_{j}=1$ and $N_{j}, M_{j} \leq 0, u_{j}=v, v_{j}=u$ if $\epsilon_{j}=-1$ for $j \in[1, k]$. By Lemma 3.8 of [32, to prove that $w(p) \in C D R(\mathbb{Z}[t], X)$ it suffices to show that

$$
g^{-1} * w(p) * g \in C D R(\mathbb{Z}[t], X)
$$

for some $g \in R(\mathbb{Z}[t], X)$.

Without loss of generality we assume $\epsilon_{k}=1$ and consider two cases. 
(i) $g_{k+1} * g_{1} * u_{1}^{N_{1}} \notin B$ or $g_{k+1} * g_{1} * u_{1}^{N_{1}} \in B$, but $\epsilon_{1}=1$.

Without loss of generality we assume the former and $\epsilon_{1}=1$. That is, $u_{1}=u, v_{1}=v$. By Lemma 20 there exists $N \in \mathbb{N}$ such that

$$
\begin{gathered}
\left(u^{-m} *\left(g_{1} * u^{N_{1}}\right)^{-1}\right) * w(p) *\left(\left(g_{1} * u^{N_{1}}\right) * u^{m}\right)=\left(u^{-N_{1}-m} * s * v^{-M_{1}}\right) \\
\circ\left(v^{M_{1}} * g_{2} * u_{2}^{N_{2}}\right) \circ \cdots \circ\left(u^{-N_{k}} * s * v^{-M_{k}-m}\right) \circ\left(v^{M_{k}+m} * g_{k+1} * g_{1} * u^{N_{1}+N}\right) \circ u^{m-N}
\end{gathered}
$$

for any $m>N$. Thus,

$\left(u^{-m} *\left(g_{1} * u^{N_{1}}\right)^{-1}\right) * w(p) *\left(\left(g_{1} * u^{N_{1}}\right) * u^{m}\right) \in C R(\mathbb{Z}[t], X) \subset C D R(\mathbb{Z}[t], X)$.

(ii) $g_{k+1} * g_{1} \in B$ and $\epsilon_{1}=-1$.

Thus, $a=s *\left(g_{k+1} * g_{1}\right) * s^{-1} \in A$ and we have

$$
\begin{gathered}
\left(g_{1} * s\right)^{-1} * w(p) *\left(g_{1} * s\right)=\left(\left(g_{2} * u_{2}^{N_{2}}\right) \circ\left(u_{2}^{-N_{2}} * s^{\epsilon_{2}} * v_{2}^{-M_{2}}\right)\right. \\
\left.\circ \cdots \circ\left(u_{k-1}^{-N_{k-1}} * s^{\epsilon_{k-1}} * v_{k-1}^{-M_{k-1}}\right) \circ\left(v_{k-1}^{M_{k-1}} * g_{k}\right)\right) * a=\left(g_{2} * u_{2}^{N_{2}}\right) \\
\circ\left(u_{2}^{-N_{2}} * s^{\epsilon_{2}} * v_{2}^{-M_{2}}\right) \circ \cdots \circ\left(u_{k-1}^{-N_{k-1}} * s^{\epsilon_{k-1}} * v_{k-1}^{-M_{k-1}^{\prime}}\right) \circ\left(v_{k-1}^{M_{k-1}^{\prime}} *\left(g_{k} * a\right)\right),
\end{gathered}
$$

where $M_{k-1}^{\prime} \in \mathbb{Z}$ is the power which works for $g_{k} * a$. So the number of $s^{ \pm 1}$ is reduced by two and we can use induction.

Now we are ready to prove the main results of this subsection from which Theorem 8 follows.

\section{Theorem 9. Put}

$$
P=P(H, s)=\left\{g * s^{\epsilon} * h \mid g, h \in H, \epsilon \in\{-1,0,1\}\right\} \subseteq C D R(\mathbb{Z}[t], X) .
$$

Then the following hold:

(1) $P$ generates a subgroup $H^{*}$ in $C D R(\mathbb{Z}[t], X)$.

(2) $P$, with the multiplication * induced from $R(\mathbb{Z}[t], X)$, is a pregroup, and $H^{*}$ is isomorphic to $U(P)$.

(3) $H^{*}$ is isomorphic to $G=\left\langle H, z \mid z^{-1} A z=B\right\rangle$.

Proof. We need the following claims.

Claim 1. Let $g_{j} * s^{\epsilon_{j}} * h_{j} \in P, j=1,2$. If

$$
g_{1} * s^{\epsilon_{1}} * h_{1}=g_{2} * s^{\epsilon_{2}} * h_{2}
$$

then $\epsilon_{1}=\epsilon_{2}$ and $h_{1} * h_{2}^{-1} \in A$ if $\epsilon_{1}=-1$ and $h_{1} * h_{2}^{-1} \in B$ if $\epsilon_{1}=1$.

To prove the claim consider an $s$-form

$$
a=\left(g_{1}, s^{\epsilon_{1}}, h_{1} * h_{2}^{-1}, s^{-\epsilon_{2}}, g_{2}^{-1}\right) .
$$

By Lemma 21, $w(a)$ is defined and

$$
g_{1} * s^{\epsilon_{1}} * h_{1} * h_{2}^{-1} * s^{-\epsilon_{2}} * g_{2}^{-1}=\varepsilon .
$$

Hence, $a$ is not reduced, and the claim follows.

For every $p \in P$ we now fix a representation $p=g_{p} * s^{\epsilon_{p}} * h_{p}$, where $g_{p}, h_{p} \in$ $H, \epsilon_{p} \in\{-1,0,1\}$.

Claim 2. Let $p=g_{p} * s^{\epsilon_{p}} * h_{p}, \quad q=g_{q} * s^{\epsilon_{q}} * h_{q}$ be in $P$. If $p * q \in P$, then either $\epsilon_{p} \epsilon_{q}=0$ or $\epsilon_{p}=-\epsilon_{q} \neq 0$ and $h_{p} * g_{q} \in A$ if $\epsilon_{p}=-1$, and $h_{p} * g_{q} \in B$ if $\epsilon_{q}=1$.

Let $x^{-1}=p * q \in P$ and $x=g_{x} * s^{\epsilon_{x}} * h_{x}$. Assume that $\epsilon_{p} \epsilon_{q} \neq 0$. 
(a) $\epsilon_{x} \neq 0$.

Consider an $s$-form

$$
a=\left(g_{p}, s^{\epsilon_{p}}, h_{p} * g_{q}, s^{\epsilon_{q}}, h_{q} * g_{x}, s^{\epsilon_{x}}, h_{x}\right) .
$$

By Lemma 21] $w(a)$ is defined and

$$
w(a)=g_{p} * s^{\epsilon_{p}} * h_{p} * g_{q} * s^{\epsilon_{q}} * h_{q} * g_{x} * s^{\epsilon_{x}} * h_{x}=\varepsilon .
$$

Hence, $a$ is not reduced and either a subsequence

$$
\left\{s^{\epsilon_{p}}, h_{p} * g_{q}, s^{\epsilon_{q}}\right\}
$$

or a subsequence

$$
\left\{s^{\epsilon_{q}}, h_{q} * g_{x}, s^{\epsilon_{x}}\right\}
$$

is reducible. In the former case we are done, so assume that $\left\{s^{\epsilon_{q}}, h_{q} *\right.$ $\left.g_{x}, s^{\epsilon_{x}}\right\}$ can be reduced. Without loss of generality we can assume that $\epsilon_{q}=-1, \epsilon_{x}=1, h_{q} * g_{x} \in A$. Hence,

$$
s^{\epsilon_{q}} * h_{q} * g_{x} * s^{\epsilon_{x}}=g \in B
$$

and we have

$$
w(a)=g_{p} * s^{\epsilon_{p}} * h_{p} * g_{q} * g * h_{x}=\varepsilon .
$$

Now, it follows that $\epsilon_{p}=0$, a contradiction with our assumption.

(b) $\epsilon_{x}=0$.

Hence, $x=g \in H$ and we consider an $s$-form

$$
a=\left(g_{p}, s^{\epsilon_{p}}, h_{p} * g_{q}, s^{\epsilon_{q}}, h_{q} * g\right) .
$$

By Lemma 21 $w(a)$ is defined and

$$
w(a)=g_{p} * s^{\epsilon_{p}} * h_{p} * g_{q} * s^{\epsilon_{q}} * h_{q} * g=\varepsilon .
$$

Now, the claim follows automatically.

Below we call a tuple $y=\left(y_{1}, \ldots, y_{k}\right) \in P^{k}$ a reduced $P$-sequence if $y_{j} * y_{j+1} \notin P$ for $j \in[1, k-1]$. Observe that if $y=\left(y_{1}, \ldots, y_{k}\right)$ is a reduced $P$-sequence and $y_{j}=g_{j} * s_{i}^{\epsilon_{j}} * h_{j}$, then either $k \leq 1$ or $y$ has the following properties which follow from Claim 2:

(a) $\epsilon_{j} \neq 0$ for all $j \in[1, k]$,

(b) if $\epsilon_{j}=-1, \epsilon_{j+1}=1$, then $h_{j} * g_{j+1} \notin A$ for $j \in[1, k-1]$,

(c) if $\epsilon_{j}=1, \epsilon_{j+1}=-1$, then $h_{j} * g_{j+1} \notin B$ for $j \in[1, k-1]$.

In particular, the $s$-form over $H$,

$$
p_{y}=\left(g_{1}, s^{\epsilon_{1}}, h_{1} * g_{2}, s^{\epsilon_{2}}, \ldots, h_{n-1} * g_{n}, s^{\epsilon_{k}}, h_{n}\right),
$$

is reduced.

To prove (1) observe first that $P^{-1}=P$. Now if $y_{1}, \ldots, y_{k} \in P$, then $y_{1} * \cdots * y_{k}=$ $w\left(p_{y}\right)$, where $y=\left(y_{1}, \ldots, y_{k}\right)$. Hence, by Lemma 21, the product $y_{1} * y_{2} * \cdots * y_{k}$ is defined in $C D R(\mathbb{Z}[t], X)$, and it belongs to $C D R(\mathbb{Z}[t], X)$. It follows that $H^{*}=\langle P\rangle$ is a subgroup of $C D R(\mathbb{Z}[t], X)$ which consists of all words $w(p)$, where $p$ ranges through all possible $s$-forms over $H$. Hence, (1) is proved.

Now we prove (2). By Theorem 2 of [38, to prove that $P$ is a pregroup and the inclusion $P \rightarrow H^{*}$ extends to an isomorphism $U(P) \simeq H^{*}$, it is enough to show that all reduced $P$-sequences representing the same element have the same $P$-length. 
Suppose two reduced $P$-sequences

$$
\left(u_{1}, u_{2}, \ldots, u_{k}\right),\left(v_{1}, v_{2}, \ldots, v_{n}\right)
$$

represent the same element $g \in H^{*}$. That is,

$$
\left(u_{1} * \cdots * u_{k}\right) *\left(v_{1} * \cdots * v_{n}\right)^{-1}=\varepsilon .
$$

We use induction on $k+n$ to show that $k=n$. Observe that $k=0$ implies $n=0$, otherwise we get a contradiction with Lemma 21(3). Hence, we can assume $k, n>0$, that is, $k+n \geq 2$. If the $P$-sequence

$$
a=\left(u_{1}, \ldots, u_{k}, v_{n}^{-1}, \ldots, v_{1}^{-1}\right)
$$

is reduced, then the underlying $s$-form is reduced, and hence, by Lemma 21 (3),

$$
w(a)=u_{1} * \cdots * u_{k} * v_{n}^{-1} * \cdots * v_{1}^{-1} \neq \varepsilon .
$$

Hence,

$$
\left(u_{1}, \ldots, u_{k}, v_{n}^{-1}, \ldots, v_{1}^{-1}\right)
$$

is not reduced and $u_{k} * v_{n}^{-1} \in P$. If $u_{k}=g_{1} * s^{\epsilon_{1}} * h_{1}, v_{n}=g_{2} * s^{\epsilon_{2}} * h_{2}$, where $g_{i}, h_{i} \in H$ and $\epsilon_{i} \in\{-1,0,1\}, i=1,2$, then by Claim 2 either $\epsilon_{1} \epsilon_{2}=0$ or $\epsilon_{1}=\epsilon_{2} \neq 0$ and $h_{1} * h_{2}^{-1} \in A$ if $\epsilon_{1}=-1$, and $h_{1} * h_{2}^{-1} \in B$ if $\epsilon_{1}=1$. In the former case, for example, if $\epsilon_{2}=0$, then $n=1, v_{n} \in H$ and $b=\left(u_{1}, \ldots, u_{k} * v_{n}^{-1}\right)$ is a reduced $P$-sequence such that $w(b)=\varepsilon$ - a contradiction with Lemma 21 (3) unless $k=1, u_{1} \in H$. In the latter case, $u_{k} * v_{n}^{-1} \in H$, and it follows that

$$
\left(u_{1}, u_{2}, \ldots, u_{k-1} *\left(u_{k} * v_{n}^{-1}\right)\right),\left(v_{1}, v_{2}, \ldots, v_{n-1}\right)
$$

represent the same element in $H^{*}$ while the sum of their lengths is less than $k+n$, so the result follows by induction.

Finally, to prove (3) observe first that $H$ embeds into $G$. We denote this embedding by $\theta$. Now we define a map $\phi: P \rightarrow G$ as follows. For $g * s^{\epsilon} * h \in P$ put

$$
g * s^{\epsilon} * h \stackrel{\phi}{\rightarrow} \theta(g) z^{\epsilon} \theta(h) .
$$

It follows from Claim 2 that $\phi$ is a morphism of pregroups. Since $H^{*} \simeq U(P)$, the morphism $\phi$ extends to a unique homomorphism $\psi: H^{*} \rightarrow G$. We claim that $\psi$ is bijective. Indeed, observe first that $G=\langle H, z\rangle$. Now, since $\psi\left(s^{\epsilon}\right)=z^{\epsilon}$ and $\psi=\phi=\theta$ on $H$, it follows that $\psi$ is onto. To see that $\psi$ is one-to-one it suffices to notice that if

$$
\left(g_{1} * s^{\epsilon_{1}} * h_{1}, g_{2} * s^{\epsilon_{2}} * h_{2}, \ldots, g_{m} * s^{\epsilon_{m}} * h_{m}\right)
$$

is a reduced $P$-sequence, then

$$
y=\left(g_{1}, s^{\epsilon_{1}}, h_{1} * g_{2}, s^{\epsilon_{2}}, \ldots, s^{\epsilon_{m}}, h_{m}\right)
$$

is a reduced $s$-form and $w(y)^{\psi} \neq 1$ by Britton's Lemma (see, for example, [29]). This proves that $\psi$ is an isomorphism, as required.

Theorem 10. Let $G=\left\langle H, z \mid z^{-1} A z=B\right\rangle$. Then, in the notation above, the free length function on $L: G \rightarrow \mathbb{Z}^{n+1}$ induced by the isomorphism $\psi: H^{*} \rightarrow G$ is regular. 
Proof. Observe that it is enough to show that the length function induced on $H^{*}=$ $\langle P\rangle$ from $C D R(\mathbb{Z}[t], X)$ is regular.

Let $g, h \in H^{*}$. Then $g$ and $h$ can be written in the unique normal forms

$$
\begin{gathered}
g=g_{1} \circ\left(u_{1}^{-N_{1}} * s^{\epsilon_{1}} * v_{1}^{-M_{1}}\right) \circ g_{2} \circ \cdots \circ\left(u_{k}^{-N_{k}} * s^{\epsilon_{k}} * v_{k}^{-M_{k}}\right) \circ g_{k+1}, \\
h=h_{1} \circ\left(w_{1}^{-L_{1}} * s^{\delta_{1}} * x_{1}^{-P_{1}}\right) \circ h_{2} \circ \cdots \circ\left(w_{m}^{-L_{m}} * s^{\delta_{m}} * x_{m}^{-P_{m}}\right) \circ h_{m+1},
\end{gathered}
$$

where $N_{j}, M_{j} \geq 0, u_{j}=u, v_{j}=v$ if $\epsilon_{j}=1$, and $N_{j}, M_{j} \leq 0, u_{j}=v, v_{j}=u$ if $\epsilon_{j}=-1$ for $j \in[1, k]$ and where $L_{i}, P_{i} \geq 0, w_{i}=u, x_{i}=v$ if $\delta_{i}=1$, and $L_{i}, P_{i} \leq 0, w_{i}=v, x_{i}=u$ if $\delta_{i}=-1$ for $i \in[1, m]$. Moreover, $g_{1}$ does not have $u_{1}^{ \pm 1}$ as a terminal subword, $g_{j}$ does not have $u_{j}^{ \pm 1}$ as a terminal subword for every $j \in[2, k], g_{j}$ does not have $v_{j-1}^{ \pm 1}$ as an initial subword for every $j \in[2, k], g_{k+1}$ does not have $v_{k}^{ \pm 1}$ as an initial subword, $h_{1}$ does not have $w_{1}^{ \pm 1}$ as a terminal subword, $h_{i}$ does not have $w_{i}^{ \pm 1}$ as a terminal subword for every $i \in[2, m], h_{i}$ does not have $x_{j-1}^{ \pm 1}$ as an initial subword for every $i \in[2, m]$, and $h_{m+1}$ does not have $x_{m}^{ \pm 1}$ as an initial subword.

If there exist $k_{1}, k_{2}>0$ such that

$$
c=c(g, h) \leq \min \left\{\left|g_{1} \circ u_{1}^{k_{1}}\right|,\left|h_{1} \circ w_{1}^{k_{2}}\right|\right\},
$$

then $\operatorname{com}(g, h)=\operatorname{com}\left(g_{1} \circ u_{1}^{k_{1}}, h_{1} \circ w_{1}^{k_{2}}\right) \in H$. Now, assume that $r \in[1, k]$ is the minimal natural number such that $\operatorname{com}(g, h)$ is an initial subword of

$$
f_{1}=g_{1} \circ\left(u_{1}^{-N_{1}} * s^{\epsilon_{1}} * v_{1}^{-M_{1}}\right) \circ g_{2} \circ \cdots \circ\left(u_{r}^{-N_{r}} * s^{\epsilon_{r}} * v_{r}^{-M_{r}}\right) \circ g_{r+1} \circ u_{r}^{p_{1}},
$$

where $p_{1} \in \mathbb{Z}$. Similarly, assume that $q \in[1, m]$ is the minimal natural number such that $\operatorname{com}(g, h)$ is an initial subword of

$$
f_{2}=h_{1} \circ\left(w_{1}^{-L_{1}} * s^{\delta_{1}} * x_{1}^{-P_{1}}\right) \circ h_{2} \circ \cdots \circ\left(w_{q}^{-L_{q}} * s^{\delta_{q}} * x_{q}^{-P_{q}}\right) \circ h_{q+1} \circ w_{q}^{p_{2}},
$$

where $p_{2} \in \mathbb{Z}$. From uniqueness of normal forms it follows that $r=q$ and that we have $g_{i}=h_{i}, u_{i}=w_{i}, v_{i}=x_{i}, N_{i}=L_{i}, \epsilon_{i}=\delta_{i}, i \in[1, r]$ and $M_{i}=P_{i}, i \in$ $[1, r-1]$.

Without loss of generality we can assume $\epsilon_{r}=1$. Hence, $v_{r}=x_{r}=v$.

Observe that $\operatorname{com}(g, h)$ can be represented as a concatenation $\operatorname{com}(g, h)=c_{1} \circ c_{2}$, where

$$
c_{1}=g_{1} \circ\left(u_{1}^{-N_{1}} * s^{\epsilon_{1}} * v_{1}^{-M_{1}}\right) \circ g_{2} \circ \cdots \circ\left(u^{-N_{r}} * s * v^{-l}\right)
$$

and $l \geq \max \left\{M_{r}, P_{r}\right\}$, and

$$
c_{2}=\operatorname{com}\left(v^{l-M_{r}} \circ g_{r+1} \circ u_{r}^{p_{1}}, v^{l-P_{r}} \circ h_{r+1} \circ w_{r}^{p_{2}}\right) .
$$

Obviously, $c_{1} \in H^{*}$. Also, $c_{2} \in H$ since $v^{l-M_{r}} \circ g_{r+1} \circ u_{r}^{p_{1}}, v^{l-P_{r}} \circ h_{r+1} \circ w_{r}^{p_{2}} \in H$, and the length function on $H$ is regular. Hence, $\operatorname{com}(g, h) \in H^{*}$.

\section{REFERENCES}

[1] R. Alperin and H. Bass, Length functions of group actions on $\Lambda$-trees. Combinatorial group theory and topology (Ed. S. M. Gersten and J. R. Stallings), Annals of Math. Studies, 111, 265-378. Princeton University Press, 1987. MR895622 (89c:20057)

[2] R. Alperin and K. Moss, Complete trees for groups with a real length function. J. London Math. Soc. (2) 31, 1985, 55-68. MR810562 (87f:20051)

[3] H. Bass, Groups acting on non-arhimedean trees. In: Arboreal group theory, MSRI Publications, 19 (1991), Springer-Verlag, New York, 69-130. MR.1105330(93d:57003)

[4] M. Bestvina and M. Feighn, Stable actions of groups on real trees. Invent. Math., 121 no. 2 (1995), 287-321. MR1346208 (96h:20056) 
[5] I. Chiswell, Abstract length functions in groups. Math. Proc. Cambridge Philos. Soc., 80 no. 3 (1976), 451-463. MR0427480 (55:512)

[6] I. Chiswell, Introduction to $\Lambda$-trees. World Scientific, 2001. MR1851337 (2003e:20029)

[7] I. Chiswell, A-free groups and tree-free groups. Algorithms, Languages, Logic (Ed. A. Borovik), Contemp. Math., Amer. Math. Soc., 378, 2005, 37-77. MR2159315(2006d:20046)

[8] B. Fine, G. Rosenberger, and M. Stille, Nielsen Transformations and Applications: A survey. Groups Korea 94, de Gruyter, 1995, 69-105. MR.1476950 (98g:20039)

[9] B. Fine, A.G. Myasnikov, V. Rebel, and G. Rosenberger, A Classification of Conjugately Separated Abelian, Commutative Transitive, and Restricted Gromov One-Relator Groups, Results in Mathematics 50, Birkhauser Verlag Basel/Switzerland, 2007, 183-193. MR 2343587 (2008k:20066)

[10] D. Gaboriau, G. Levitt and F. Paulin, Pseudogroups of isometries of $\mathbb{R}$ and Rips' Theorem on free actions on $\mathbb{R}$-trees. Israel. J. Math., 87 (1994), 403-428. MR.1286836 (95e:20042)

[11] A. Gaglione, B. Fine, A.G. Myasnikov, G. Rosenberger, and D. Spellman. A classification of fully residually free groups of rank three or less. J. of Algebra, 200 no. 2 (1998), 571-605. MR.1610668 (99b:20053)

[12] A. M. W. Glass, Partially ordered groups. Series in Algebra, 7, World Scientific, 1999. MR1791008 (2001g:06002)

[13] V. Guirardel, Limit groups and groups acting freely on $\mathbb{R}^{n}$-trees. Geom. Topol., 8 (2004), 1427-1470. MR2119301 (2005m:20060)

[14] N. Harrison, Real length funtions in groups. Trans. Amer. Math. Soc., 174 (1972), 77-106. MR0308283 (46:7397)

[15] A. H. M. Hoare, On length functions and Nielsen methods in free groups. J. London Math. Soc. (2), 14 (1976), 188-192. MR0422420 (54:10409)

[16] A. H. M. Hoare, Nielsen method in groups with a length function. Math. Scand., 48 (1981), 153-164. MR631332 (83a:20041)

[17] I. Kapovich and R. Weidmann, Two-generated groups acting on trees. Archiv der Mathematik, 73 no. 3 (1999), 172-181. MR.1705011(2001d:20022)

[18] I. Kapovich and R. Weidmann, Nielsen methods and groups acting on hyperbolic spaces. Geometriae Dedicata 98 (2003), 95-121. MR 1988426 (2004e:20076)

[19] B. Khan, A.G. Myasnikov and D. Serbin, On positive theories of groups with regular free length functions. Internat. J. Algebra Comput., 17 no. 1 (2007), 1-26. MR2300402 (2008g:20067)

[20] O. Kharlampovich, A.G. Myasnikov, Irreducible affine varieties over a free group. II: Systems in triangular quasi-quadratic form and description of residually free groups. J. of Algebra 200 no. 2 (1998), 517-570. MR1610664 (2000b:20032b)

[21] O. Kharlampovich, A.G. Myasnikov, Implicit function theorems over free groups. J. of Algebra, 290 no. 1 (2005), 1-203. MR2154989 (2007b:20047)

[22] O. Kharlampovich and A.G. Myasnikov Effective JSJ decompositions. Contemp. Math. series of the AMS, Algorithms, Languages, Logic (Borovik, ed.), 378 (2005), 87-212. MR2159316 (2006m:20045)

[23] O. Kharlampovich, A. Myasnikov, V. Remeslennikov, D. Serbin, Subgroups of fully residually free groups: Algorithmic problems, Contemp. Math. series of the AMS, Group theory, Statistics and Cryptography 360 (2004), 63-101. MR2105437 (2006b:20046)

[24] O. Kharlampovich, A. Myasnikov, and D. Serbin, Regular actions on $\Lambda$-trees. Preprint.

[25] V. Kopytov and N. Medvedev, Right-ordered groups. Siberian School of Algebra and Logic. Consultants Bureau, New York, 1996. MR1393199 (97h:06024a)

[26] R. Lyndon, Length functions in groups. Math. Scand., 12 (1963), 209-234. MR0163947 $(29: 1246)$

[27] R. Lyndon and P. Schupp, Combinatorial group theory. Ergebnisse der Mathematik und ihrer Grenzgebiete, 89, Springer-Verlag, Berlin, Heidelberg, New York, 1977. MR0577064 $(58: 28182)$

[28] G.S. Makanin, Equations in a free group. (Russian), Izv. Akad. Nauk SSSR, Ser. Mat., 46 (1982), 1199-1273 (transl. in Math. USSR Izv. 21 (1983)). MR682490 (84m:20040)

[29] W. Magnus, A. Karrass and D. Solitar, Combinatorial group theory: Presentations of groups in terms of generators and relators. Dover Publications, New York, 1976. MR0422434 $(54: 10423)$ 
[30] J. Morgan and P. Shalen, Valuations, Trees, and Degenerations of Hyperbolic Structures, I. Annals of Math, 2nd Ser., 120 no. 3. (1984), 401-476. MR769158 (86f:57011)

[31] A. Myasnikov, V. Remeslennikov, Length functions on free exponential groups. Proc. $N 26$. IITPM SO RAN, Omsk, 1996, 1-34.

[32] A. Myasnikov, V. Remeslennikov and D. Serbin, Regular free length functions on Lyndon's free $\mathbb{Z}[t]$-group $F^{\mathbb{Z}[t]}$, Algorithms, Languages, Logic (Ed. A. Borovik), Contemp. Math., Amer. Math. Soc., 378 (2005), 37-77. MR2159314 (2006e:20057)

[33] A. Myasnikov, V. Remeslennikov and D. Serbin, Fully residually free groups and graphs labeled by infinite words, Internat. J. Algebra and Comput., 16 (2006), no. 4, 689-737. MR2258835 (2007k:20053)

[34] A. Nikolaev and D. Serbin, Finite index subgroups of fully residually free groups. Preprint 2009.

[35] D. Promislow, Equivalence classes of length functions on groups. Proc. London Math. Soc (3) 51 (1985), 449-477. MR805717 (87e:20063)

[36] A. Razborov, On systems of equations in a free group. Math. USSR Izvestiya, 25 no. 1 (1985), 115-162. MR755958 (86c:20033)

[37] F.S. Rimlinger, Pregroups and Bass-Serre Theory. Mem. Amer. Math. Soc., Providence, 65, no. 361 (1987). MR874086 (88i:20046)

[38] F.S. Rimlinger, A subgroup theorem for pregroups. Combinatorial group theory and topology (Alta, Utah, 1984), 163-174, Ann. of Math. Stud. 111, Princeton Univ. Press, Princeton, NJ, 1987. MR895615 (88g:20053)

[39] J.-P. Serre, Trees. New York, Springer, 1980. MR607504 (82c:20083)

[40] J.R. Stallings, Group theory and three dimensional manifolds. New Haven, London, Yale Univ. Press, 1971. MR0415622 (54:3705)

[41] R. Weidmann, On the rank of amalgamated products and product knot groups. Mathematische Annalen, 312 no. 4 (1998), 761-771. MR.1660235 (99k:20052)

[42] R. Weidmann, The Nielsen method for groups acting on trees, Proc. London Math. Soc., 85 no. 1 (2002), 93-118. MR 1901370 (2003c:20029)

Department of Mathematics and Statistics, Hunter College CUNy, 695 Park Avenue, New York, New York 10065

Department of Mathematical Sciences, Stevens Institute of Technology, 1 Castle Point on Hudson, Hoboken, New Jersey 07030

Department of Mathematics, Omsk State University, 55-A Prospect Mira, Omsk, RusSIA 644077

Department of Mathematical Sciences, Stevens Institute of Technology, 1 Castle Point on Hudson, Hoboken, New Jersey 07030 